\title{
Diffuse elastic waves in a nearly axisymmetric body: Energy distribution, transport and dynamical localization
}

\author{
Richard L. Weaver ${ }^{\mathrm{a}}$, John Y. Yoritomo, and J. Patrick Coleman \\ Department of Physics, University of Illinois at Urbana-Champaign, 1110 W. Green Street, \\ Urbana, Illinois 61801, USA
}

Received 24 June 2016 / Received in final form 14 September 2016 Published online 25 May 2017

\begin{abstract}
We report measurements and theory on the distribution and evolution of diffuse ultrasonic waves in elastic bodies with weakly broken axisymmetry. Aluminum cylinders with dimensions large compared to wavelength were excited by transient point sources at the center of one of the circular faces. The resulting power spectral density PSD was then examined as a function of time and frequency and position on that face. It was found that the PSD showed a marked concentration at the center at early times, a concentration that subsequently slowly diminished towards a state of uniformity across the face, over times long compared to ultrasonic transit time across the sample. The evolution is attributed to scattering by symmetry breaking heterogeneities. Relaxation did not proceed all the way to uniformity and equipartition, behavior shown to be consistent with Enhanced Backscatter and Dynamical Anderson Localization.
\end{abstract}

\section{Review/background}

There is a long literature on diffuse ultrasound in solids with potential applications in nondestructive evaluation [1-9] and seismology [10-16]. Diffuse field ultrasound is employed also for explorations of stochastic wave fields in general, including systems described by random matrix theory and systems that may be analogs for mesoscopic electronics and quantum chaos [17-33]. Weaver [31] provides an overview and tutorial. Diffuse field concepts have been applied to room acoustics and vibro-acoustics in structures [32-34]. Throughout this work attention has generally been confined to elastic or fluid structures with little or no geometric symmetry. Irregular shapes and/or the presence of random scatterers have been imagined as required for the diffuse field assumption in which we take the field subsequent to a transient excitation to have been scattered sufficiently that the resulting wave field is maximally garbled. It is preferred to study structures with no special symmetries, so that that the energy is well mixed across all modes and positions. Ideally one would wish for structures in which ray trajectories are chaotic [28] and ergodic. Should the sample have a good

\footnotetext{
a e-mail: r-weaver@illinois.edu
} 
symmetry (say a reflection symmetry) then energy originally deposited in one symmetry state (e.g even) is never scattered into the other (e.g., odd). In consequence the diffuse field is a superposition of two or more nonmixing diffuse fields and lacks full equipartition. Examples of this are provided by Weaver [22] and Ellegaard et al. [23] who showed that symmetry in solid blocks had a significant effect on the statistics of the eigenfrequencies.

In recent years, diffuse elastic waves have found application in seismology. Multiply scattered seismic coda waves at wavelengths of the order of kms approach the condition of full diffusivity $[10,11]$. Seismic noise generated by ocean storms (with wavelengths of the order of tens of $\mathrm{km}$ ) is not well scattered before being dissipated, but nevertheless shares some of the characteristics of fully diffuse waves. Correlations of such noise fields have been found to permit retrieval of approximate seismic Greens functions, without the need for controlled sources [12,13,35-37]. At longer seismic wavelengths yet one would imagine that diffuse field analysis would not apply; the earth appears spherically symmetric on long length scales. To the extent that the earth is exactly spherically symmetric, seismic waves will preserve their wave angular momentum. Waves associated with different vector spherical harmonics will not mix and the usual diffuse field arguments would not apply [38]. To the extent that the actual earth weakly breaks spherical symmetry, spherical harmonics will mix. One therefore anticipates the possibility that a long wavelength seismic diffuse field will make a transition from a superposition of many independent diffuse fields corresponding to different vector spherical harmonics to a single global field. The rate of that transition should be a measure of the degree of symmetry breaking, with longer wavelengths mixing more slowly.

With a view towards informing diffuse field analysis in axisymmetric structures in general and for long wavelength coda waves in the earth in particular (we envision an examination of the coda of 100 second waves from large earthquakes with ring down times up to of days (e.g., [16,38]) here we study the ultrasonic diffuse field dynamics of nearly axisymmetric laboratory bodies. The next two sections describe our laboratory system and some of the diffuse field measurements made there. Section 3 presents a simple model for expected behaviors, whose predictions are then shown to be not in accord with measurements. Section 4 then develops a more precise theory for diffuse waves in nearly axisymmetric bodies. It begins by developing theory for modal densities and mean square surface displacements in subspaces of specified angular momentum. (We use the term angular momentum to describe the azimuthal Fourier index; it directly corresponds to true angular momentum for quantum mechanical but not for classical waves.) A time-domain statistical energy like model for the incoherent migration of energy between angular momentum subspaces is proposed that predicts evolution towards a state of equipartition in which all angular momenta have the same energy per mode. It is then argued that enhanced backscatter and dynamical Anderson localization can modify that prediction and affect late time energy densities, and in particular, prevent equipartition. It is demonstrated that the parameters of our system are such that dynamical localization should be strong. These ideas are then used in Section 5 to analyze the laboratory measurements and confirm their consistency with theory.

\section{Laboratory system}

Our samples are aluminum cylinders of thickness $L$ of the order of $100 \mathrm{~mm}$ and diameters $2 R$ of $176 \mathrm{~mm}$. Our wavefields are composed of ultrasonic elastic waves at frequencies between 300 and $950 \mathrm{kHz}$, corresponding to shear wavelengths between 9 and $3 \mathrm{~mm}$ respectively - short compared to cylinder dimensions. Quality factors $Q$ 
Digital Scope/ PC

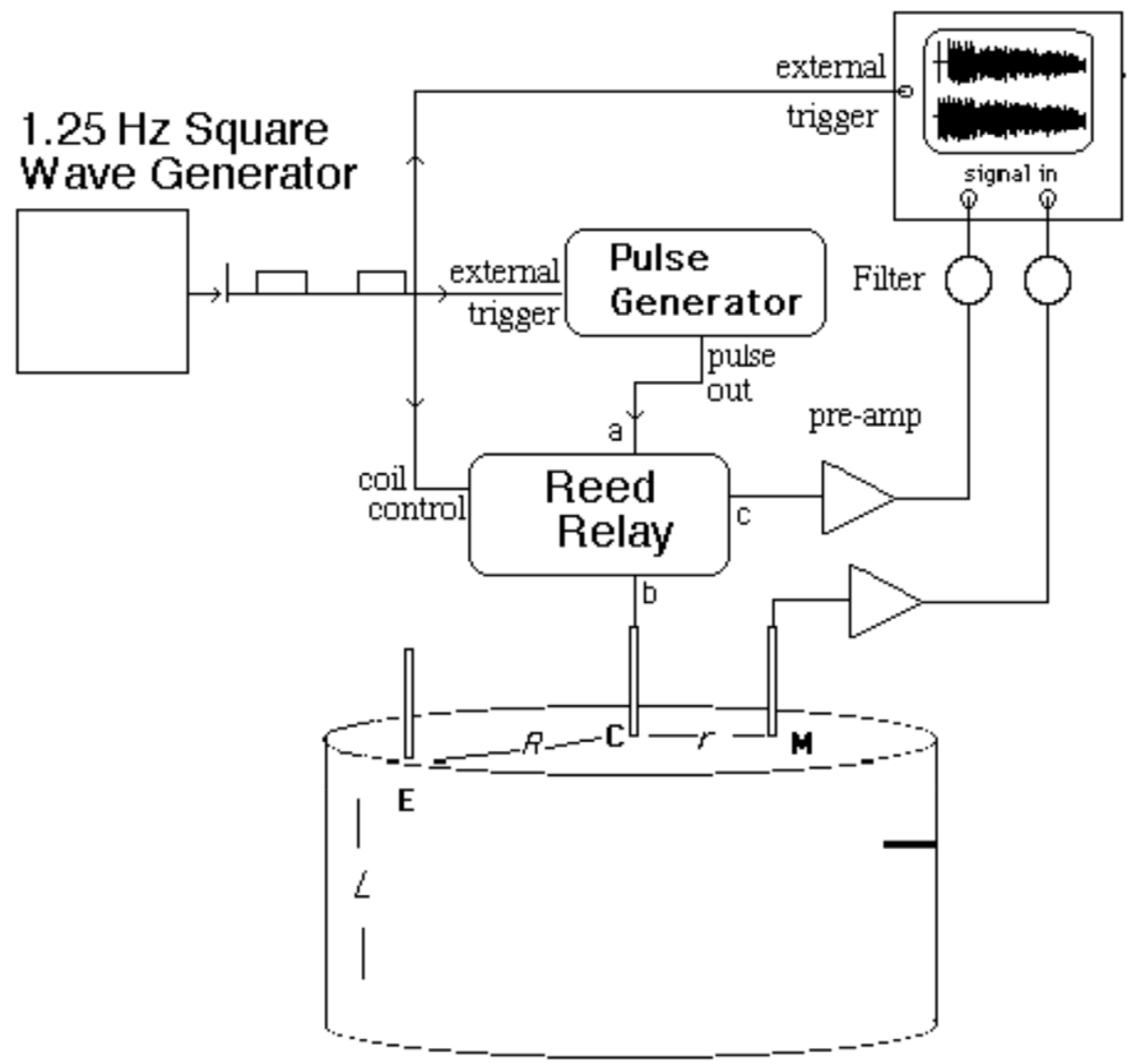

Fig. 1. Schematic of experimental system. An 6061 polycrystalline aluminum alloy cylinder of radius $R=88 \mathrm{~mm}$ and height $L=100 \mathrm{~mm}$ is prepared by saw and lathe, then polished and cleaned to reduce surface losses. A voltage pulse was applied to the center transducer and the resulting field was detected at the center and termed signal ' $C C$.' The response at a point $\mathrm{M}$ at $r=50 \mathrm{~mm}$ was also collected, and termed ' $C M$.' Also shown is a transducer near the edge ' $E$ ' used as an alternate source. A $12 \mathrm{~mm}$ deep hole of $4 \mathrm{~mm}$ diameter is drilled (and tapped) normally into the outer surface a distance $55 \mathrm{~mm}$ from the bottom. The cylinder was supported on three upward-pointing wide-headed $2 \mathrm{~cm}$ nails, with point diameters about $0.4 \mathrm{~mm}$, in turn resting on a rubber sheet.

are greater than $10^{5}$, thus permitting study of diffuse field evolution over long times usually sufficient to ascertain the asymptotic behavior of the energy distribution.

\subsection{Samples and transducers}

A cylinder of interest is pictured in Figure 1. It has radius $R=88 \mathrm{~mm}$ and height $L=100 \mathrm{~mm}$. The pictured cylinder has a symmetry-breaking $12 \mathrm{~mm} \times 4 \mathrm{~mm}$ hole tapped into its outer surface. A Valpey-Fisher VP-1063 thin ultrasonic "pin" transducer (1.6 mm diameter, $30 \mathrm{~mm}$ length, sensitive to vertical motion) is placed (without the usual liquid couplant and with a minimal contact force of 45 grams i.e., $0.44 \mathrm{~N}$ ) 
at the center ' $C$ ' of the top face. Another (VP-1093, $2.36 \mathrm{~mm}$ diameter, also sensitive to vertical motion) is placed (also without couplant but with a contact force of $13 \mathrm{~N}$ ) at a point midway ' $M$ ' between edge and center a distance $50 \mathrm{~mm}$ from the center and yet another VP-1093 at point ' $E$ ' $78 \mathrm{~mm}$ from the center. The use of couplant and/or greater weights would improve signal to noise ratios, but the associated inhomogeneous losses due to the corresponding greater transducer intrusiveness would distort the diffuse field energy evolution and complicate attempts to make quantitative predictions. For this reason we use dry coupling.

\subsection{Sources and signal acquisition}

We wish to measure wave response at $\mathrm{C}$ and at $\mathrm{M}$ due to a source at $\mathrm{C}$. Diffuse signals detected at a source can be problematic, because at late times a diffuse field can be so weak due to dissipation that even low noise that may leak from the pulse generator into the detection circuit can compete with the desired signal. To avoid this, we use a reed relay (see Fig. 1). On receipt of the $5 \mathrm{~V}$ square wave coil control signal the reed relay detaches from its rest position in which port a is connected to port $\mathrm{b}$ and commences to make a connection between ports $b$ and $c$. The process of de-connection and re-connection takes about 200 microseconds. Thus the generator's pulse (of 10 nsec duration) proceeds without hindrance through the reed to excite the source transducer at C. Two hundred microseconds later the reed has detached from $a$ and made the connection between $b$ and $c$. At these later times the signal detected by $\mathrm{C}$ will reach its preamplifier, and do so without contamination from pulser noise. The signal is then anti-alias filtered and digitized. $400 \mathrm{msec}$ later when the square wave control signal drops back to zero the reed returns to its rest position connecting $a$ and $b$. The process repeats every $800 \mathrm{msec}$. Signals received at both C and M, are amplified (by $60 \mathrm{~dB}$ for $\mathrm{C}$ and $40 \mathrm{~dB}$ for $\mathrm{M}$, regardless of whether the source was at $\mathrm{C}$ or at E), four-pole anti-alias low-pass filtered with $3 \mathrm{~dB}$ points at 2250 and $950 \mathrm{kHz}$ respectively, and digitized at 16 bits and $5 \mathrm{MSa} / \mathrm{sec}$ for $100 \mathrm{msec}$.

The first few msec of a typical signal detected at the center transducer due to a source at the center is shown in Figure 2. An approximately $300 \mu$ sec interval starting at $t=0$ is not meaningful, as the reed relay mechanism leaves the transducer unconnected, or poorly connected, to its receiver circuit during that period. To compensate for the poor signal to noise caused by the dry coupling, the signal has been repetition averaged 1000 times, taking about 34 minutes.

\subsection{Minimizing temperature drifts}

The specimen has been enclosed under a plastic dome in order to limit temperature fluctuations. A temperature change $\Delta \mathrm{T}$ is known to lead to an average fractional wavespeed change of $\mathrm{C} \Delta T$ where $\mathrm{C}$ in aluminum is $0.027 \%$ per degree Celsius $[26,39]$. This in turn leads to a phase shift at frequency fof $2 \pi f t \mathrm{C} \Delta T$ where t is the time since the source impulse acted. At a frequency $f$ the varied phase shifts will lead to destructive interferences and diminishment of perceived power by a factor

$$
\left|\frac{1}{\Delta T} \int_{\Delta T} d T \exp (2 \pi i f t C T)\right|^{2}=\left|\frac{\sin (\pi f t C \Delta T)}{\pi f t C \Delta T}\right|^{2} .
$$

A thermocouple monitors the temperature during the repetition process and allows us to confirm that temperature fluctuations are not great enough to cause phase differences at late times that would significantly degrade the average. Temperature 


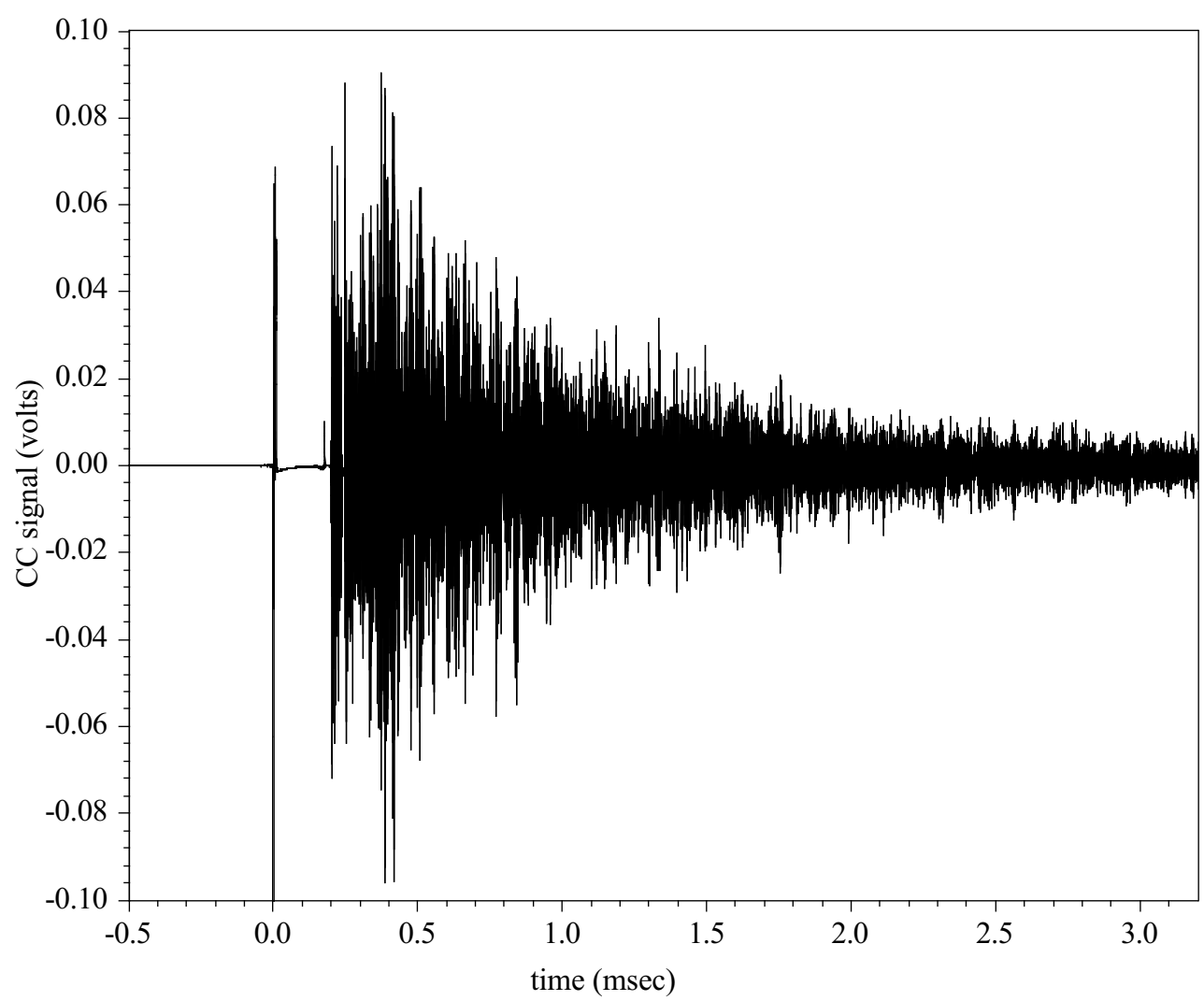

Fig. 2. The first few msec of signal CC. The main bang is visible at time zero. The first 300 microsec after that are not meaningful and are not used in the analysis.

changes were less than $\Delta T=0.05$ degrees Celsius across the duration of the measurement (the figure is limited by our thermocouple's precision; we actually expect it was better than that because the temperature was constant to within $0.1 \mathrm{C}$ over a period of hours, much longer than the 34 minutes required for 1000 repetitions). The above estimate then indicates that degradation due to temperature fluctuations is small (certainly no more than $50 \%$ at $f=500 \mathrm{kHz}$ after $t=100 \mathrm{msec}$ ). In any case, such degradation only diminishes signal-to-noise ratios uniformly for all signals and would not impact the behavior seen in ratios of energies that we wish to investigate. Furthermore, it will be seen that the data show no evidence of non-exponential decays like those predicted by the above equation.

\subsection{Spectra}

A short section of the Fourier transform (absolute value of FFT) of the signal CM is shown in Figure 3. The rapid Erickson fluctuations on a fine scale of $\delta f \sim 20 \mathrm{~Hz}$ with amplitudes of the order of $100 \%$ seen in $3 \mathrm{~b}$ are characteristic of any stochastic signal (and for which correlation range $\delta f$ scales inversely with signal duration here identifiable as a combination of inverse dissipation rate and the record length $100 \mathrm{msec}$ ) and are not due to electronic noise or transducer resonances. Nor are they individual normal modes of the structure, these are not well resolved here. 


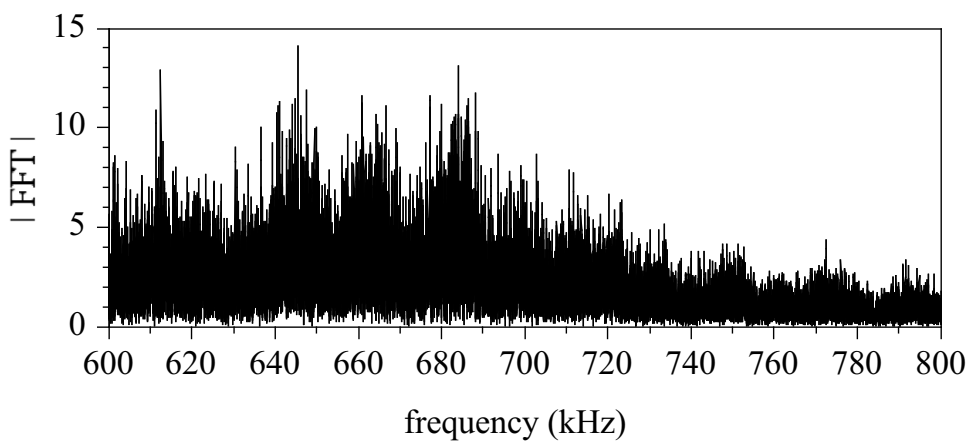

(a)

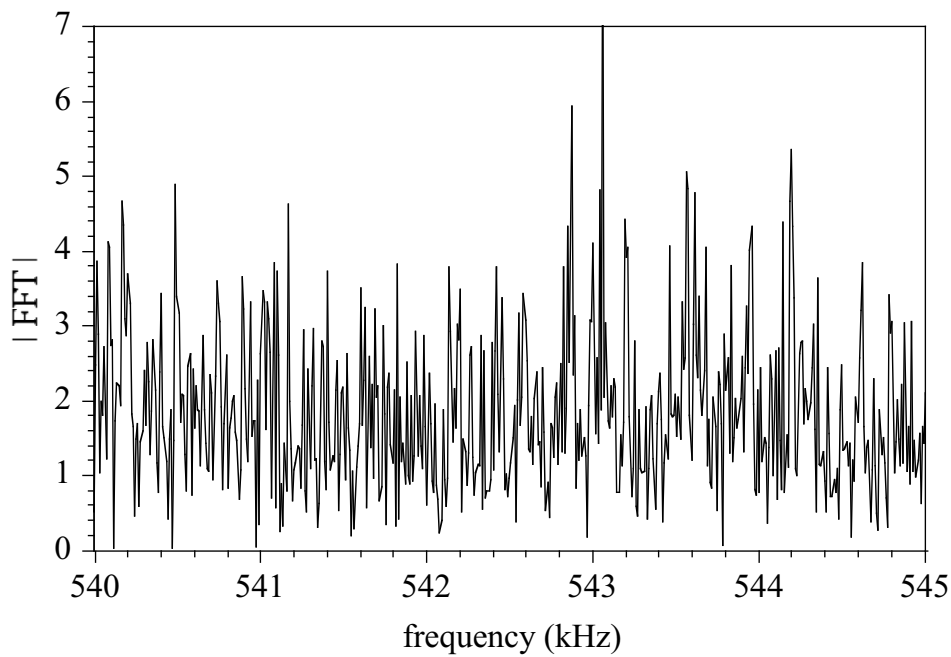

(b)

Fig. 3. a) A short section of the spectrum of signal CM. b) The spectrum on a fine scale.

The longer range variations seen in $3 \mathrm{a}$ on a scale of $20 \mathrm{kHz}$ are, we conjecture, due to reverberations through the thickness or across the surface with periods of order $2 \mathrm{~L} / c_{s} \sim 2 \mathrm{R} / c_{s} \sim 50 \mu$ sec. Longer range variations are sometimes observed also; these are due to reverberant reflections over the $30 \mathrm{~mm}$ length of the transducers, with round trip travel times of the order of $20 \mu \mathrm{sec}$. For many applications in ultrasonics the latter irregularity is undesirable (and transducer designers seek to minimize them), because the corresponding time-domain distortions disperse otherwise distinct ray arrivals. In diffuse field applications without discernable ray arrivals they are less objectionable, while the pinducers' low intrusiveness is desirable. Hence our choice of these pin transducers.

\subsection{Pinducer intrusiveness}

We measured the intrusiveness of the thin pinducer used at $C$ by a method analogous to methods used in room acoustics to measure absorptivity of surface panels or solid objects. The pinducer was placed - without couplant and with 45 grams of contact force $(0.44 \mathrm{~N})$ - on an irregular block of volume $1095 \mathrm{~cm}^{3}$, insonified and measured respectively by two other transducers. This placement was found to increase the rate of 
energy dissipation by 0.00055 factors of e of energy per msec (mostly independent of frequency in our range). Thus we characterize the pinducer's absorption by the quantity $p=0.6 \mathrm{~cm}^{3} / \mathrm{msec}$. If we divide by a characteristic wave speed $c_{s}=310 \mathrm{~cm} / \mathrm{msec}$ ( $c_{s}$ because shear waves dominate a diffuse elastic wave field [27]), this can be converted to an absorption cross section of $0.0019 \mathrm{~cm}^{2}$, one tenth the geometric cross section of the face of a $1.6 \mathrm{~mm}$ diameter cylinder: $0.02 \mathrm{~cm}^{2}$. Clearly the majority of the wave energy incident upon the transducer is not absorbed into it. This quantity $p$ will be used in the analyses of Section 5.

\section{Power spectral densities CC and CM and their time-evolution}

Many diffuse field analyses measure and model the evolution of wave spectral energy density in space and time (e.g., [4,19-21,31]). For the cylinder of Figure 1 we anticipate the evolution to be described by the following picture, informed chiefly by the assumed almost fully preserved axisymmetry. Under the idealization that the axisymmetry is perfect, all normal modes of the structure will have definite angular momentum, as quantified by an azimuthal index $m$ that takes negative and positive integer values. The index $m$ plays a role in the mode shape, such that the components $\left\{\mathrm{u}_{\mathrm{r}}, \mathrm{u}_{\mathrm{z}}, \mathrm{u}_{\theta}\right\}$ of the vector field vary with angle like $\exp (\mathrm{i} m \theta)$. The transient normal force generated in the transducer $\mathrm{C}$ creates bulk $\mathrm{P}$ and $\mathrm{SV}$ waves. It also generates Rayleigh waves that constitute the majority, $\sim 64 \%$, of the deposited energy $[40,41]$. If the normal point force source is on the axis of the cylinder, then all waves created have $m=0$. On a transit time scale $\sim 2 \mathrm{R} / c_{s}$ (which here is about 50 $\mu \mathrm{sec}$ ) these waves reach the edge and mode convert to other $\mathrm{P}$ and SV and Rayleigh waves. Within the assumption that the body is exactly axisymmetric, they retain their vanishing angular momentum even as they mode convert. After the waves have experienced a few such wall and edge reflections we anticipate that a fully diffuse P-SV-Rayleigh $m=0$ field is established (it will lack $m=0$ torsional SH waves). This field will have much less Rayleigh wave energy and more bulk wave energy than the initial deposition. The conversion of the Rayleigh waves is due to scattering at the edges. According to Gautesan [42] for Poisson ratio 0.33, on a Rayleigh wave encountering an edge about $50 \%$ of the energy is transmitted to the vertical surface and about $11 \%$ is reflected. The remainder is mode converted to bulk waves. The Rayleigh energy on the top surface will therefore diminish with each reflection by $89 \%$ until it asymptotes at a proportion characteristic of an $m=0$ fully diffuse field. That proportion is given by the ratio of modal densities of $\mathrm{P} / \mathrm{SV}$ bulk to Rayleigh waves, which is of order $\left(\mathrm{L} \omega / \pi c_{s}\right)$. The field will therefore need something of order $\ln \left(\mathrm{L} \omega / \pi c_{s}\right) /|\ln (11 \%)|$ transits for the initial Rayleigh wave energy to mix with all the $\mathrm{P} / \mathrm{SV}$ waves at $m=0$ and fill the depth uniformly. A few hundred microseconds should more than suffice in the bodies used here.

On a longer time scale ( $\sim 5$ msec in these experiments, see below) the waves will start sampling whatever non-axisymmetry the structure may have. Our initial $m=0$ diffuse $\mathrm{P} / \mathrm{SV} / \mathrm{R}$ waves can mode convert to $m \neq 0 \mathrm{P} / \mathrm{SV} / \mathrm{R}$ waves, also to $\mathrm{SH}$ waves, and to the $m=0 \mathrm{SH}$ waves that were not excited by the original source. At $m \neq 0$ all $\mathrm{SH}$ and $\mathrm{P} / \mathrm{SV} / \mathrm{R}$ waves of the same $m$ mode convert to each other and equilibrate on fast transit time scales, so we lump them together into separate diffuse fields, one for each $m$.

Our interest is in studying the spatial distribution of the wave energy, and its evolution as the waves sample the weak non-axisymmetry. The preeminent observable feature of the initial distribution is anticipated to be a deficit of power spectral density at $\mathrm{M}$ relative to that at $\mathrm{C}$ by a factor of the order $r$ times wavenumber 
where $r$ is the distance of $\mathrm{M}$ from the center. At short time (much less than 5 msec) the field is all in the $m=0$ subspace and so vertical displacement $u_{z}$ varies with $r$ like some superposition of Bessel functions of different radial wavenumbers $\sim \mathrm{J}_{\mathrm{o}}(\xi \mathrm{r})$ where $\xi$ is a wave number of order $2 \pi f / c_{s}$. On recalling the asymptotic form $\mathrm{J}_{\mathrm{o}} \sim(2 / \pi \xi \mathrm{r})^{1 / 2} \cos (\xi \mathrm{r}-\pi / 4)$, one concludes that the mean square normal displacement at the center should be enhanced over that at larger $r$ by a factor of $\pi \xi r$. (It is further enhanced by an enhanced backscatter EBS factor of two or three $[14,18,19,43]$, of which more in Sect. 4.5 below.) This rough argument is made more precise in Section 4.2 where it is shown why edge effects may be neglected and how $\xi$ should be interpreted.

The diffuse field established before the first msec as purely $m=0$ should then, as it samples any nonaxisymmetry and in particular as it scatters off the hole in the side, evolve. The $m=0$ subspace should lose energy to other $m$. It is useful to first imagine evolution under the simplifying assumption of incoherence, with no Anderson localization and no enhanced backscatter. We also assume no significant differences in absorption amongst the various subspaces. With those assumptions one concludes that at late enough times the energies at all values of $m$ will have equilibrated and the field will have become globally diffuse; all points on the surface becoming equivalent (except for some possible variations close to the edge) such that the energy at the center is no longer enhanced over the energy at other distances. So, regardless of the character and rate of the transport amongst angular momentum states (as long as there $i s$ transport) we predict a substantial change in energy profile across the top surface. The ratio (mean square signal at $\mathrm{M}$ due to a source at the center $\mathrm{C}$ divided by mean square signal at $\mathrm{C}$ due to the same source) is predicted to increase over time by a factor of order $\pi \xi r$. In the cylinders used here and at the frequencies examined here, and for $r=50 \mathrm{~mm}$, this factor is over a hundred and potentially dramatic.

Figure 4 shows the power spectral density (PSD) in the $390-468 \mathrm{kHz}$ frequency band versus time for the signal received at $C$ due to the source at $C$ (labeled ' $C C$ ') and the signal simultaneously received at $M$ for the same source (labeled ' $C M$ '). (It also shows PSDs due to a source at $E$, these will be discussed in Sect. 5.) Repetition averaged signals like that pictured in Figure 2 were time-windowed into coterminous windows of duration $\Delta t=0.8192 \mathrm{msec}$ (excluding the interval from 0 to $300 \mu \mathrm{sec}$ ), Fourier transformed, squared, and summed into several non-overlapping frequency bands of width $\Delta f=78 \mathrm{kHz}$. The figure shows the time dependence of the PSDs in one such band. The noise level is seen at negative times. The first non-negative time is centered on $0.709 \mathrm{msec}$ corresponding to the window that spans times between $t=300$ and $1119 \mu \mathrm{sec}$; successive time windows are spaced by $\Delta t=0.8192 \mathrm{msec}$. The familiar approximately exponential decay of the energy ascribed $[1,2,24,31]$ in whole or part to ultrasonic absorption is readily seen. Each data point is subject to some irreducible fluctuation due to the stochastic nature of the signal and not related to electronic noise. Standard arguments [44] assuming stationarity across $\Delta t$ and whiteness across $\Delta f$ teach that the natural logarithm of each binned energy has a standard deviation statistical error of $(\Delta t \Delta f)^{-1 / 2}=0.125$.

The two transducers have, intrinsically and also due to their different contact forces, different sensitivities to vertical displacements (they also have different filters and amplifiers) so the two power spectral densities cannot be compared directly. Nevertheless we can examine the evolution of their ratio CC/CM, predicted above to diminish by a factor $\pi \xi r$ about equal to 100, as time proceeds from zero to infinity. Examination of the data in Figure 4 shows the ratio of these power spectral densities diminishes by a factor of about 12. Diminishment of the ratio is apparent, but is much less than anticipated. This is a chief result. 


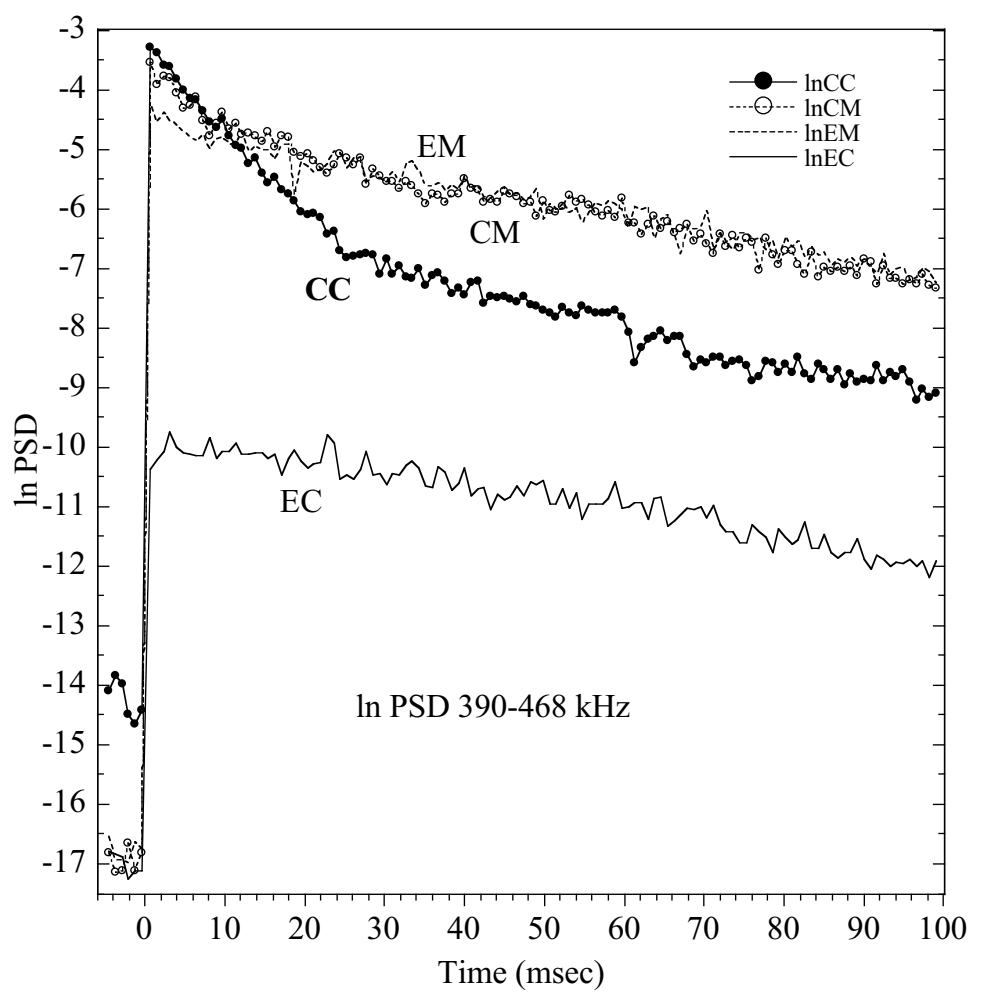

Fig. 4. The power spectral densities at $\mathrm{C}$ and $\mathrm{M}$ in the $390-468 \mathrm{kHz}$ frequency bin due to sources at $\mathrm{C}$ and at $\mathrm{E}$. $\mathrm{CC}=$ source at $\mathrm{C}$, receiver at $\mathrm{C} ; \mathrm{CM}=$ source at $\mathrm{C}$, receiver at $\mathrm{M} ; \mathrm{EC}=$ source at $\mathrm{E}$, receiver at $\mathrm{C} ; \mathrm{EM}=$ source at $\mathrm{E}$, receiver at $\mathrm{M}$. $\mathrm{CC}$ decays at early times like $\exp (-\lambda t)$ with $\lambda=0.18 / \mathrm{msec}$. At late times all PSD decay like $\exp (-\sigma t)$ with $\sigma=0.025 / \mathrm{msec}$. The ln PSD fluctuate, with theoretical standard deviation 0.125.

The next section develops a diffuse field theory with which to predict these profiles, their ratios, and how they vary in time. We will find that the above picture based on incoherent evolution, though attractively simple, is overly simple. The actual distribution does not relax to equipartition and uniformity, even at infinite time. The picture can be made more quantitatively precise. It must also be corrected to account for enhanced backscatter and for dynamical Anderson localization.

\section{Theory for angular-momentum resolved diffuse waves}

The normal modes and responses of a thick solid cylinder with traction-free boundaries, even if axisymmetry is unbroken, are known only numerically. Hutchinson [45] discusses the modes when wavelengths are comparable to cylinder size. Here we address the statistics of higher frequency modes and responses. Theory to date for diffuse elastic waves has not considered fields of specified angular momentum. Hence we begin by revisiting some basics. Key questions concern modal density in $m$-specific subspaces. We also require estimates for the participation of each angular momentum in the mean square normal displacement on the top surface and the closely related question as to how a specified source distributes its radiated energy in $m$. This section 
concludes with a theory for the way in which energy migrates between $m$-specific subspaces, and with a discussion of how Anderson localization and enhanced backscatter modify that picture.

\subsection{Modal densities}

Key to diffuse field analysis is the concept of modal density. Conventional arguments indicate that the mode count $N$ (number of modes with frequencies below $\omega$ ) of a scalar wave of speed $c$ in a volume $V$ is given to leading order at short wavelength by the first term of the Weyl series [46]

$$
N(\omega)=\frac{V}{\pi^{3}} \int_{\text {first octant }} d^{3} k \Theta\left(\omega / c-\sqrt{k_{x}^{2}+k_{y}^{2}+k_{z}^{2}}\right)=\frac{V}{\pi^{3}} \frac{1}{8} \frac{4 \pi}{3 c^{3}} \omega^{3}=\frac{V \omega^{3}}{6 c^{3} \pi^{2}}
$$

obtained by associating one mode to each unit volume in $\mathbf{k}$-space $(\Delta k)^{3} V / \pi^{3}$ and integrating over the first octant in a Cartesian k-space. $\Theta$ is the Heaviside function, equal to unity if the argument is positive and zero otherwise. The formula is extended to elastic waves by summing $N$ over two distinct $S$ waves and one $P$ wave $[27,47]$.

$$
N_{\text {total }}=\frac{V \omega^{3}}{6 \pi^{2}}\left[2 c_{s}^{-3}+c_{p}^{-3}\right]
$$

Rayleigh waves are relatively negligible at leading order in mode counting, contributing only terms of order $S \omega^{2} / c^{2}$ where $S$ is the surface area. Contributions to $N$ of that smaller order are usually neglected. They depend on boundary conditions $[22,48]$. Modal density $n$ is the $\omega$ derivative of $N ; n=\partial N / \partial \omega$.

The same count may be attempted by summing over angular momentum states and integrating over radial and axial wavenumbers $k_{r}$ and $k_{z}$ in cylindrical coordinates rather than Cartesian.

$$
N=\frac{L}{\pi} \int_{k_{z}>0} d k_{z} \sum_{m=-\infty_{k_{r}>0}}^{\infty} \frac{d k_{r}}{\Delta k_{r}} \Theta\left(\omega / c-\sqrt{k_{r}^{2}+k_{z}^{2}}\right) .
$$

The spacing $\Delta k_{r}$ in radial wave number is not constant as it is for a Cartesian wavenumber, but rather $\Delta k_{r} \sim(\pi / R)\left[1-m^{2} / k_{r}^{2} R^{2}\right]^{-1 / 2}$. See Watson [49] section 15.82. This estimate is obtained by examining the spacing of the zeros of Bessel functions $J_{m}(z)$. For $z \gg m$, the zero spacings are $\pi$; for $z$ close to but larger than $m$ they are greater than $\pi$. Of course for $z<m$ there are no zeros except for the trivial zero at $z=0$, so the square root $\left[1-m^{2} / k_{\mathrm{r}}^{2} R^{2}\right]^{1 / 2}$ is interpreted as zero when $|m|>k_{r} R$.

Therefore

$$
N=\frac{L R}{\pi^{2}} \int_{k_{z}>0} d k_{z} \sum_{m=-\infty_{k_{r}>0}}^{\infty} d k_{r} \sqrt{1-m^{2} / k_{r}^{2} R^{2}} \Theta\left(\omega / c-\sqrt{k_{r}^{2}+k_{z}^{2}}\right) .
$$

The sum over $m$ may be converted to an integral (valid for large enough $k_{\mathrm{r}} R$ )

$$
\sum_{m=-\infty}^{\infty} \sqrt{1-m^{2} / k_{r}^{2} R^{2}} \approx 2 \int_{0}^{k_{r} R} \sqrt{1-m^{2} / k_{r}^{2} R^{2}} d m=k_{r} R \pi / 2 .
$$


The mode count then becomes

$$
\begin{aligned}
N & =\frac{L R^{2}}{2 \pi} \int_{k_{z}>0} d k_{z} \int_{k_{r}>0} k_{r} d k_{r} \Theta\left(\omega / c>\sqrt{k_{r}^{2}+k_{z}^{2}}\right) \\
& =\frac{L R^{2}}{2 \pi} \int_{k_{r}>0} k_{r} d k_{r} \sqrt{\omega^{2} / c^{2}-k_{r}^{2}} \\
& =\frac{L R^{2}}{2 \pi c^{3}} \omega^{3} \int_{0}^{1} \sqrt{1-x^{2}} x d x=\frac{L R^{2}}{6 c^{3} \pi} \omega^{3}=\frac{V \omega^{3}}{6 c^{3} \pi^{2}}
\end{aligned}
$$

which agrees with the conventional Cartesian derivation above.

We may adapt the above approach to calculate the number of modes $N_{m}$ in subspace $m:(m=-\infty \ldots 0,1, \ldots \infty)$

$$
\begin{aligned}
N_{m} & =\frac{L R}{\pi^{2}} \int_{k_{z}>0} d k_{z} \int_{k_{r}>0} d k_{r} \sqrt{1-m^{2} / k_{r}^{2} R^{2}} \Theta\left(\omega / c>\sqrt{k_{r}^{2}+k_{z}^{2}}\right) \\
& =\frac{L R}{\pi^{2}} \int_{k_{r}>0} d k_{r} \sqrt{1-m^{2} / k_{r}^{2} R^{2}} \sqrt{\omega^{2} / c^{2}-k_{r}^{2}} \\
& =\frac{L R}{c^{2} \pi^{2}} \omega^{2} \int_{\arcsin (|m| c / R \omega)}^{\pi / 2} \cos ^{2} \theta d \theta \sqrt{1-c^{2} m^{2} / \omega^{2} R^{2} \sin ^{2} \theta} \\
& =\frac{L R}{4 c^{2} \pi} \omega^{2}[1-|m| c / R \omega]^{2} \Theta(R|\omega| / c-|m|) .
\end{aligned}
$$

It is apparent that $m$-resolved modal density diminishes with $m$ and vanishes for $|m|>R \omega / c$.

At $m=0$, it is

$$
N_{0}=\frac{L R}{4 \pi c^{2}} \omega^{2}
$$

Similarly, the sum of $N_{\mathrm{m}}$, over all $m$ from $-\infty$ to $\infty$, is merely $N$ itself, equal to $\mathrm{LR}^{2} \omega^{3} / 6 \mathrm{c}^{3} \pi$. The modal densities are the $\omega$ derivatives of these:

$$
\begin{aligned}
n_{0} & =\frac{L R}{2 \pi c^{2}} \omega \\
n_{m} & =\frac{L R}{2 \pi c^{2}}(\omega-|m| c / R) \Theta(\omega-|m| c / R) \\
2 \sum_{m>0} n_{m} & =\frac{d}{d \omega}\left(N-N_{0}\right) \approx \frac{d}{d \omega} \frac{L R^{2} \omega^{3}}{6 \pi}=\frac{L R^{2} \omega^{2}}{2 \pi c^{3}} .
\end{aligned}
$$

In the short wavelength limit at $\omega R / c \gg 1$, the $m=0$ modes constitute a small fraction of the total.

Of course in a homogeneous isotropic elastic body, these modal densities must be summed over two shear waves and one dilatational wave.

For our particular $100 \mathrm{~mm}$ high by $88 \mathrm{~mm}$ radius aluminum cylinder, we take $c_{\mathrm{S}}=3.1 \mathrm{~mm} / \mu \mathrm{sec}$ and $c_{\mathrm{p}}=6.2 \mathrm{~mm} / \mu \mathrm{sec}$. We calculate $n_{o}$ (not counting $\left.\mathrm{SH}\right)=$ $\mathrm{dN}_{0} / \mathrm{d} \omega=1.145 \mathrm{msec}$ times frequency in $\mathrm{MHz}(f / \mathrm{MHz}), n_{\mathrm{o}}^{\mathrm{SH}}=0.916 \mathrm{msec}(f / \mathrm{MHz})$. Total modal density $n_{\text {total }}=\mathrm{d} N / \mathrm{d} \omega$ is $347(f / \mathrm{MHz})^{2}$ msec. 


\subsection{Mean square field amplitudes}

Diffuse field analysis makes the assumption that all modes of similar frequency have statistically equal energy $\varepsilon$ per mode. For a diffuse field that is resolved in $m$, and well mixed within each $m$-subspace, and for which there is only slow transport between different $m$, we make the generalized assumption that all modes at specified $m$ have energy $\varepsilon_{m}$ per mode, a quantity that may differ for different $m$. Slow time-evolution of the $\varepsilon_{m}$ may take place due to scattering between the weakly coupled subspaces identified with different $\mathrm{m}$; this is discussed in subsection 4.4.

A measurement like that described in Section 2 of free vibration dynamic displacement $\psi_{\nu}$ (in direction $\nu$ and band limited to a frequency range $\Delta \omega$ ) at a position $\mathbf{r}$ in a generic solid may be modeled in terms of the normal modes

$$
\psi_{\nu}(\boldsymbol{r}, t)=R e \sum_{\text {modes } s}^{\prime} a_{s} u_{\nu}^{(s)}(\boldsymbol{r}) \exp \left(i \omega_{s} t\right)
$$

where $\mathbf{u}^{(s)}$ is the $s^{t h}$ mode (normalized such that $\int \rho\left|\mathbf{u}^{s}(\boldsymbol{r})\right|^{2} d V=1$ where $\rho$ is mass density) and $\omega_{\mathrm{s}}$ is its natural frequency. The prime on the sum indicates that the sum is confined to those modes $s$ with frequency within a range $\Delta \omega$ in the vicinity of some central frequency. The factor $a_{\mathrm{s}}$ is the mode's amplitude. The energy in that mode is $\varepsilon=\omega_{s}^{2}\left|a_{s}\right|^{2} / 2$. The diffuse field assumption in which mode amplitudes are uncorrelated and each mode in the vicinity of a given central frequency has equal expected energy translates to each $a_{s}$ having equal mean square value $\left\langle a_{\mathrm{s}} a_{s^{\prime}}^{*}\right\rangle=2 \delta_{\mathrm{ss}^{\prime}} \varepsilon / \omega_{\mathrm{s}}^{2}$. The brackets \langle\rangle may be understood as an ensemble average. Thus, where $n$ is modal density, the mean square displacement $U$ (at position $r$, in direction $\nu$, integrated over the frequency band and over the ensemble) is

$$
U \equiv\left\langle\psi_{\nu}(\boldsymbol{r}, t)^{2}\right\rangle=\left(\varepsilon / \omega^{2}\right) n \Delta \omega\left\langle u_{\nu}^{(s)}(\boldsymbol{r})^{2}\right\rangle .
$$

The average indicated by brackets \langle\rangle on the right side may now be considered an average over all modes $s$ in the range $\Delta \omega$, and using an ergodicity assumption understood to apply to a typical specimen from the ensemble.

The mean square mode shape at $\boldsymbol{r},\left\langle u_{\nu}^{(s)}(\boldsymbol{r})^{2}\right\rangle$, that appears above may [40] be computed from a calculation of the Green function at $\boldsymbol{r}$. This may be seen in the following argument: Consider the modal sum representation of the frequency domain Green's dyadic ( $\eta$ is an infinitesimal positive quantity introduced to ensure causality)

$$
\overleftrightarrow{G}\left(\boldsymbol{r}, \boldsymbol{r}^{\prime}, \omega\right)=\sum_{s} \frac{\boldsymbol{u}^{(s)}(\boldsymbol{r}) \boldsymbol{u}^{(s)}\left(\boldsymbol{r}^{\prime}\right)}{\omega_{s}^{2}-(\omega-i \eta)^{2}}
$$

The imaginary part of a diagonal element, eg. $\operatorname{Im} G_{\nu \nu}$, at $\boldsymbol{r}=\boldsymbol{r}^{\prime}$ is related to the rate at which a harmonic point force (in the $\nu$-direction, at $\boldsymbol{r}$ ) does work. More importantly here, it is (using the familiar expression from the calculus of distributions [50] $(x-(y-i \eta))^{-1}=P(x-y)^{-1}-i \pi \delta(x-y), \mathrm{P}$ being Cauchy principle part)

$$
\operatorname{Im} G_{\nu \nu}(\boldsymbol{r}, \boldsymbol{r}, \omega)=-\frac{\pi}{2 \omega} \sum_{s} u_{\nu}^{(s)}(\boldsymbol{r})^{2} \delta\left(\omega-\omega_{s}\right)
$$

The sum is interpretable (if a factor of $\rho$ is inserted) as local density of states [51]. Equation (14) permits us to identify the mean square mode shape $\left\langle u_{\nu}^{(s)}(\boldsymbol{r})^{2}\right\rangle$ as a frequency average of $\operatorname{Im} G$ :

$$
(2 \omega / \pi) \int_{\Delta \omega} \operatorname{Im} G_{\nu \nu}(\boldsymbol{r}, \boldsymbol{r} ; \omega) d \omega=-n \Delta \omega\left\langle u_{\nu}^{(s)}(\boldsymbol{r})^{2}\right\rangle .
$$

This allows us to estimate $\left\langle u^{2}\right\rangle$ from a separate calculation of $G$. 
Finally we obtain the key result that a diffuse field distributes its mean square strength in proportion to the energy-per-mode $\varepsilon$ and to (real part of) drive point admittance.

$$
U \equiv\left\langle\psi_{\nu}(\boldsymbol{r}, t)^{2}\right\rangle=(-2 \varepsilon / \omega \pi) \int_{\Delta \omega} \operatorname{Im} G_{\nu \nu}(\boldsymbol{r}, \boldsymbol{r} ; \omega) d \omega
$$

This connection, between a diffuse field participation at a point and the Green function at that point, is the $\boldsymbol{r}=\boldsymbol{r}^{\prime}$ case of the widely derived connection between the Green function between points $\boldsymbol{r}$ and $\boldsymbol{r}^{\prime}$ and the correlations of a diffuse field between those points [35-37]. The identity has been used to interpret measurements that compare mean square diffuse seismic displacements in horizontal and vertical directions in order to infer local stratigraphy [15]. In a form similar to the above it was first derived in elastodynamics by Weaver [40], but can be found earlier in acoustics $[52,53]$. The result is derived in a Structural Acoustics context by Shorter and Langley [34], who term the relation between $\mathrm{U}$ and $\mathrm{G}$ diffuse field reciprocity.

We now wish to extend this argument to the case in which the field may have different energies $\varepsilon_{m}$ in different angular momentum subspaces. The modal sum for the free vibration (11) is now broken into a sum over angular momenta and a sum over modes $s$ at that angular momentum

$$
\psi_{\nu}(\boldsymbol{r}, t)=R e \sum_{m} \sum_{s \in m}^{\prime} a_{s} u_{\nu}^{(s)}(\boldsymbol{r}) \exp \left(i \omega_{s} t\right)
$$

such that the mean square diffuse field signal in the band is now

$$
\begin{aligned}
U \equiv\left\langle\psi_{\nu}(\boldsymbol{r}, t)^{2}\right\rangle & =\sum_{m}\left(\varepsilon_{m} / \omega^{2}\right) n_{m} \Delta \omega\left\langle\boldsymbol{u}_{\nu}^{(s \in m)}(\boldsymbol{r})^{2}\right\rangle \\
& =(-2 / \omega \pi) \sum_{m} \varepsilon_{m} \int_{\Delta \omega} \operatorname{Im} G_{\nu \nu}^{(m)}(\boldsymbol{r}, \boldsymbol{r} ; \omega) d \omega .
\end{aligned}
$$

It is given in terms of the m-resolved Green's function $G^{(m)}$.

In order to evaluate the above expression for $U$, we need to evaluate the frequency smoothed and $m$-resolved Green's function for the structure, in particular for points on the top surface of our cylinder and for normal directions $\nu$ where the measurements described in Sections 2 and 3 were made. Calculations of elastodynamic $G$ in a finite body are complex and usually can only be done numerically, and even there they are generally confined to low frequencies for which wavelengths are of order or greater than system size [45]. However, here it suffices to consider an unbounded half space rather than the finite cylinder of the experiments. This is because reflections from the boundaries arrive after finite time equal to the round trip travel time from the point of interest to the edge, so if our frequency averaging range $\Delta \omega$ is broad enough, the edge cannot contribute to deposited spectral energy density [54]. (This incurs some error if the point $r$ of interest is at or near the edge and $\Delta \omega$ is small.) Thus the calculation of G reduces to Lamb's problem [41] for a half space. This is a classic calculation and amenable to analytic treatment. On performing a double-spatial Fourier transform and introducing the Fourier transform vector variable $\boldsymbol{\xi}$ in the horizontal plane, the governing equations for the harmonic Greens function due to a harmonic normal point source at $\mathbf{r}^{\prime}=\left(r^{\prime}, \theta^{\prime}\right)$ on the surface become coupled ordinary differential equations in depth $z$. After solving these and performing the inverse double spatial Fourier transform for a receiver position $\mathbf{r}=(r, \theta)$ on the surface, one obtains [41]

$$
G_{z z}\left(\boldsymbol{r}, \boldsymbol{r}^{\prime}, \omega\right)=-\frac{1}{4 \mu \pi^{2}} \frac{\omega^{2}}{c_{s}^{2}} \int d^{2} \boldsymbol{\xi} \alpha \frac{\exp \left(i \boldsymbol{\xi} \cdot\left(\boldsymbol{r}-\boldsymbol{r}^{\prime}\right)\right)}{D(\boldsymbol{\xi}, \omega)}
$$


where $D$ is the Rayleigh function

$$
D(\xi, \omega)=\left(2 \xi^{2}-\omega^{2} / c_{s}^{2}\right)^{2}-4 \alpha \beta \xi^{2}
$$

and $\alpha$ and $\beta$ are (the imaginary unit $i$ times) the vertical $P$ and $\mathrm{S}$ wavenumbers associated with the horizontal wave vector $\xi$ :

$$
\alpha=\sqrt{\xi^{2}-\omega^{2} / c_{p}^{2}} ; \quad \beta=\sqrt{\xi^{2}-\omega^{2} / c_{s}^{2}}
$$

with the sign chosen such that they have positive real part. Singularities are resolved as in equation 13 by interpreting $\omega$ as $\omega-i \eta$. $\mu$ is the shear modulus.

We expand the $\mathbf{r}$ dependence in a partial wave expansion taken from Abromowitz and Stegun's [55] formulas 9.1.44 and 45.

$$
\exp (i \boldsymbol{\xi} \cdot \boldsymbol{r})=\exp \left(i \xi r \cos \left(\theta-\theta_{\xi}\right)\right)=\sum_{m=-\infty}^{\infty} i^{m} J_{m}(\xi r) e^{i m\left(\theta-\theta_{\xi}\right)}
$$

where $\theta_{\xi}$ is the direction of the vector $\boldsymbol{\xi}$. Without loss of generality we also assume the source at $r^{\prime}$ is on the $x$-axis, $\theta^{\prime}=0$, to get

$$
G_{z z}\left(\boldsymbol{r}, \mathbf{r}^{\prime} ; \omega\right)=-\frac{1}{4 \mu \pi^{2}} \frac{\omega^{2}}{c_{s}^{2}} \sum_{m=-\infty}^{\infty} \int_{0}^{\infty} \xi d \xi d \theta_{\xi} \alpha \frac{\exp \left(-i \xi r^{\prime} \cos \theta_{\xi}\right)}{D(\xi, \omega)} i^{m} J_{m}(\xi r) e^{i m\left(\theta-\theta_{\xi}\right)}
$$

The integration over $\theta_{\xi}$ may be performed:

$$
\int d \theta_{\xi} \exp \left(-i \xi r^{\prime} \cos \theta_{\xi}\right) e^{-i m \theta_{\xi}}=2 \pi i^{-m} J_{m}\left(\xi r^{\prime}\right) .
$$

One then derives, for the steady state $z$-displacement at position $r$ on the surface consequent to a unit harmonic vertical force on the surface on the $x$-axis at $r^{\prime}$

$$
G_{z z}\left(\boldsymbol{r}, \boldsymbol{r}^{\prime} ; \omega\right)=-\frac{1}{4 \mu \pi^{2}} \frac{\omega^{2}}{c_{s}^{2}} \sum_{m=-\infty}^{\infty} 2 \pi \int \xi d \xi \alpha \frac{J_{m}(\xi r)}{D(\xi, \omega)} e^{i m \theta} J_{m}\left(\xi r^{\prime}\right) .
$$

This $G_{z z}$ is the response at $r, \theta$ to a unit source at $r^{\prime}$ on the $x$ axis. The sum over $m$ is manifestly a sum over the generated waves $J_{m}(\xi r) \exp (\operatorname{im} \theta)$ at angular momentum $m$. We now specify to the response at the source position $\left(\theta=0, r=r^{\prime}\right)$ and obtain

$$
\operatorname{Im} G_{z z}(\boldsymbol{r}, \boldsymbol{r} ; \omega)=-\frac{1}{4 \mu \pi^{2}} \frac{\omega^{2}}{c_{s}^{2}} \operatorname{Im} \sum_{m=-\infty}^{\infty} 2 \pi \int \xi d \xi \alpha \frac{J_{m}^{2}(\xi r)}{D(\xi, \omega)} .
$$

So that we identify the $m$-resolved $G$ by

$$
\operatorname{Im} G_{z z}^{(m)}(\boldsymbol{r}, \boldsymbol{r} ; \omega)=-\frac{1}{4 \mu \pi^{2}} \frac{\omega^{2}}{c_{s}^{2}} 2 \pi \int_{0}^{\infty} \xi d \xi \operatorname{Im} \alpha \frac{J_{m}^{2}(\xi r)}{D(\xi, \omega)} .
$$

The integrand is positive semi-definite.

We define an overbar as a weighted average of a $\xi$ dependent quantity

$$
\bar{X} \equiv \frac{\int \xi d \xi X(\xi) \operatorname{Im} \frac{\alpha}{D(\xi, \omega)}}{\int \xi d \xi \operatorname{Im} \frac{\alpha}{D(\xi, \omega)}}
$$


to obtain

$$
\operatorname{Im} G_{z z}^{(m)}(\boldsymbol{r}, \boldsymbol{r} ; \omega)=-\frac{1}{2 \mu \pi} \frac{\omega^{2}}{c_{s}^{2}}\left[\operatorname{Im} \int \frac{\xi d \xi \alpha}{D(\xi, \omega)}\right] \overline{J_{m}^{2}(\xi r)} .
$$

We now recall equation 18 , insert $\nu=\mathrm{z}$ for normal displacement, and deduce for the mean square normal displacement at $\mathrm{r}$ integrated over the band:

$$
U=\left[\frac{\omega \Delta \omega}{\mu c_{s}^{2} \pi^{2}} \int \xi d \xi \operatorname{Im} \frac{\alpha}{D(\xi, \omega)}\right]\left[\varepsilon_{0} \overline{J_{o}^{2}(\xi r)}+2 \sum_{m>0} \varepsilon_{m} \overline{J_{m}^{2}(\xi r)}\right] .
$$

Thus the mean square diffuse signal $U$ at $\mathbf{r}$ on the top surface is expressed in terms of the $\varepsilon_{\mathrm{m}}$. Inasmuch as we are chiefly interested in $U$ 's $r$-dependence (and its time dependence via time dependence in the $\varepsilon$ ) the prefactor in square brackets is ignorable. That $U$ 's r-dependence within each m-specific subspace is $J_{m}^{2}(\xi r)$ was anticipated in Section 3.

The integral (28) that defines our overbar is dominated by the contribution from the Rayleigh pole at $\xi=\omega / c_{\text {Rayleigh }}$. The majority of energy goes into Rayleigh surface waves. Hence we might for simplicity approximate the overbar average (28) by an evaluation at $\xi=\omega / c_{R}$. A more precise value, that includes contributions from the $\mathrm{P}$ and $\mathrm{SV}$ and head waves in $\mathrm{G}$, requires a numerical evaluation of the integral. One important case is the overbar of $1 / \xi$. On defining a kind of effective wave speed $c^{*}$ by $\overline{1 / \pi \xi r}=1 / \pi \xi^{*} r=c^{*} / \pi \omega r$ (the inverse of $c^{*}$ corresponds to a kind of average radial slowness, as weighted by the efficiency with which a normal point force radiates energy, or equivalently the efficiency with which a point vertical displacement detector measures diffuse energy) we calculate

$$
\begin{aligned}
c^{*}= & \omega \int d \xi \operatorname{Im} \frac{\alpha}{D(\xi, \omega)} / \int \xi d \xi \operatorname{Im} \frac{\alpha}{D(\xi, \omega)} \\
= & c_{s} \int_{0}^{\infty} d s \operatorname{Im} \frac{\sqrt{s^{2}-K^{2}}}{\left(4 s^{2}+1\right)^{2}-4 \sqrt{s^{2}-K^{2}} \sqrt{s^{2}-1}} \\
& / \int_{0}^{\infty} s d s \operatorname{Im} \frac{\sqrt{s^{2}-K^{2}}}{\left(4 s^{2}+1\right)^{2}-4 \sqrt{s^{2}-K^{2}} \sqrt{s^{2}-1}}=1.29 c_{s}
\end{aligned}
$$

where $K$ is the ratio of wavespeeds $c_{s} / c_{p}$. The value 1.29 was obtained numerically for the case $K=\frac{1}{2}$ corresponding to aluminum with Poisson ratio $1 / 3$.

Figure 5 shows the indefinite integral of the weight function $\operatorname{Im} \xi \alpha / D$ at Poisson ratio $1 / 3$. It has been normalized to $100 \%$ at large slowness. A small imaginary part was subtracted from $\omega$ to regularize the numerics. The singular step-up at the Rayleigh slowness 1.07 is apparent. The inset shows the weight function itself, dominated by the Rayleigh singularity.

\subsection{Some special cases of $U(r)$}

\subsubsection{Equipartition over all $m$}

If all the $\varepsilon$ are equal, as they would be in a fully diffuse field, then equation 30 tells us (ignoring the prefactor)

$$
U \propto \varepsilon\left[\overline{J_{o}^{2}(\xi r)}+2 \sum_{m>0} \overline{J_{m}^{2}(\xi r)}\right]
$$




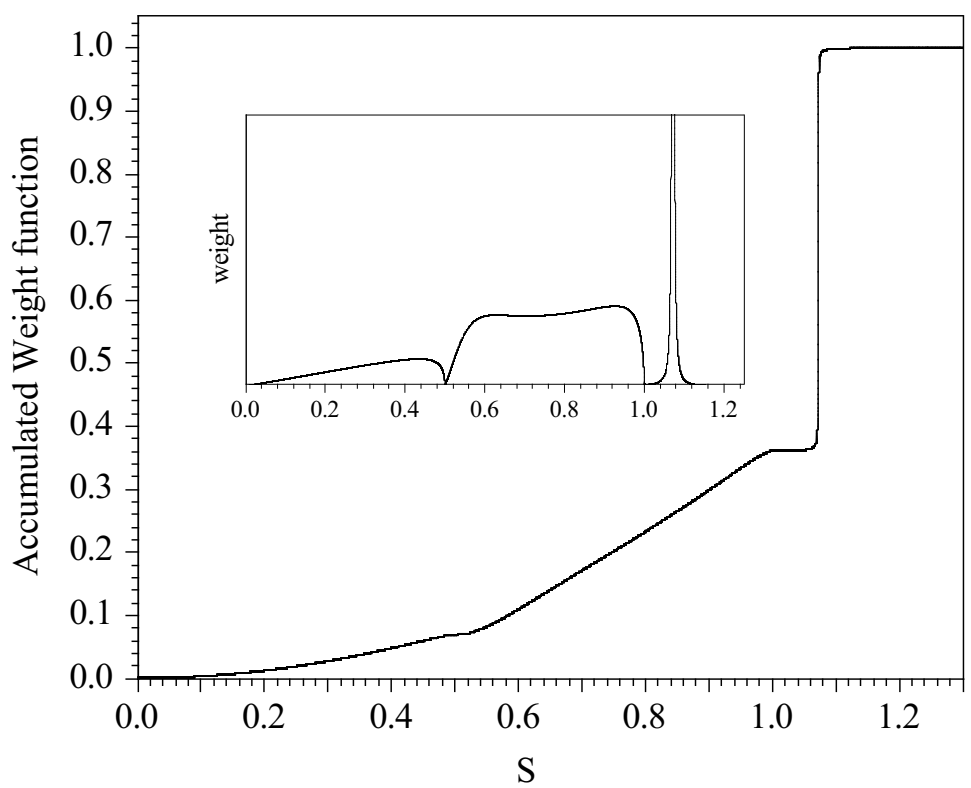

Fig. 5. The integral $\int_{0}^{S} s \sqrt{s^{2}-K^{2}} /\left\{\left(4 s^{2}+1\right)^{2}-4 \sqrt{s^{2}-K^{2}} \sqrt{s^{2}-1}\right\} d s$ of the weight function for Poisson ratio $1 / 3$ is normalized to $100 \%$ and plotted versus nondimensional slowness $S$. The singular step-up of about 0.64 is visible at the Rayleigh slowness 1.07 (indicating that $64 \%$ of the energy goes into Rayleigh waves). Other non-analyticities are also discernable, at the longitudinal slowness 0.5 and at the shear slowness 1.0. The weight function itself is portrayed in the inset, smoothed slightly to soften the Rayleigh singularity.

But, using identity 9.1.76 from Abromowitz and Stegun [55],

$$
J_{o}^{2}(\xi r)+2 \sum_{m \geq 1} J_{m}^{2}(\xi r)=1
$$

we conclude that all $\varepsilon$ equal implies that $U$ is independent of $r$, as it ought be; a fully diffuse field will distribute its mean square displacement uniformly over the top surface.

\subsubsection{Equipartition amongst all $m \neq 0$}

For the case in which the $\varepsilon_{m}$ are not all equal, general evaluation of the sum (30) is not possible except numerically. However, the special case in which all $\varepsilon_{m}$ for $m \neq 0$ are equal to each other while $\varepsilon_{0}$ differs is addressable. In this case we write,

$$
U=\left[\frac{\omega \Delta \omega}{\mu c_{s}^{2} \pi^{2}} \int \xi d \xi \operatorname{Im} \frac{\alpha}{D(\xi, \omega)}\right]\left[\varepsilon_{0} \overline{J_{o}^{2}(\xi r)}+2 \varepsilon_{m \neq 0} \sum_{m>0} \overline{J_{m}^{2}(\xi r)}\right]
$$

and use the identity (32) to replace the sum over $J_{m}^{2}$ to obtain

$$
\begin{aligned}
U & =\left[\frac{\omega \Delta \omega}{\mu c_{s}^{2} \pi^{2}} \int \xi d \xi \operatorname{Im} \frac{\alpha}{D(\xi, \omega)}\right]\left[\varepsilon_{0} \overline{J_{o}^{2}(\xi r)}+\varepsilon_{m \neq 0}\left(1-\overline{J_{0}^{2}(\xi r)}\right)\right] \\
& =\left[\frac{\omega \Delta \omega}{\mu c_{s}^{2} \pi^{2}} \int \xi d \xi \operatorname{Im} \frac{\alpha}{D(\xi, \omega)}\right]\left[\varepsilon_{m \neq 0}+\left(\varepsilon_{0}-\varepsilon_{m \neq 0}\right) \overline{J_{o}^{2}(\xi r)}\right] .
\end{aligned}
$$


At the center, $r=0$, where $J_{\mathrm{o}}=1$, this is

$$
U_{\text {center }}=\frac{\omega \Delta \omega}{\mu c_{s}^{2} \pi^{2}} \varepsilon_{0} \int \xi d \xi \operatorname{Im} \frac{\alpha}{D(\xi, \omega)} .
$$

At a distance $r$ large compared to $1 / \xi$ such that $J_{0}(\xi r)$ may be approximated as $\cos (.$. $[\pi \xi r / 2]^{-1 / 2}$ and on doing an average over a short range of $\omega$ to permit replacing $\cos ^{2}$ with $\frac{1}{2}$, it is

$$
U_{a t r \neq 0}=\left[\frac{\omega \Delta \omega}{\mu c_{s}^{2} \pi^{2}} \int \xi d \xi \operatorname{Im} \frac{\alpha}{D(\xi, \omega)}\right]\left[\varepsilon_{m \neq 0}+\left(\varepsilon_{0}-\varepsilon_{m \neq 0}\right) \overline{1 / \pi \xi r}\right] .
$$

The ratio $U_{\mathrm{r}} / U_{\text {center }}$ was discussed in Sections 2 and 3 . It is

$$
\begin{aligned}
U_{a t r \neq 0} / U_{\text {center }} & =\left[\varepsilon_{m \neq 0}+\left(\varepsilon_{0}-\varepsilon_{m \neq 0}\right) \overline{1 / \pi \xi r}\right] / \varepsilon_{0} \\
& =\overline{1 / \pi \xi r}=1 / \pi \xi^{*} r \quad \text { when } \quad \varepsilon_{m \neq 0}=0 .
\end{aligned}
$$

This ratio is unity at equipartition when $\varepsilon_{m \neq 0}=\varepsilon_{0}$, and is the small quantity $1 / \pi \xi^{*} \mathrm{r}$ when all energy is in $m=0$, as it is at early times in the measurements of Section 3 .

For the measurements of Section 3, the enhancement factor $\overline{1 / \pi \xi r}^{-1}$ is $2 \pi^{2} \mathrm{fr} / \mathrm{c}^{*}$, equal to $247(\mathrm{f} / \mathrm{MHz})$ if $r=50 \mathrm{~mm}$ using $c^{*}=1.29 c_{\mathrm{s}} . U(r)$ due to a source at the center is, before any transport between angular momentum subspaces, predicted to be strongly concentrated at the center. Thus we arrive at a confirmation of the argument of Section 3, and find that $U$ at the center should be enhanced, at early times, over $U$ at point ' $M$ ', CC/CM, by a factor of $247(f / \mathrm{MHz})$. The $\mathrm{CC}$ and $\mathrm{CM}$ data of Figure 4 at early times do not permit corroboration of this enhancement, because the relative sensitivity of the transducers is not known. As a first attempt to calibrate them it is tempting to evaluate the ratio $\mathrm{CC} / \mathrm{CM}$ at late times, and assume equipartition at those times. This argument then leads to a prediction that the ratio CC/CM that may be discerned in Figure 4 should decrease by a factor of $247(\mathrm{f} / \mathrm{MHz})=106$ from time zero to late times, much more than the observed 12 . The reason for this striking discrepancy is discussed below.

\subsubsection{Sources far from center.}

If a source acts at a point $r^{\prime}$ other than the center, the initial distribution $U(\mathrm{r})$ is more complicated. We may calculate it from equation (30) using $\varepsilon_{m}$ as given by the following argument. The energy (per frequency) deposited in angular momentum $m$ by an impulse at $r^{\prime}$ is (using Eq. (29) and neglecting $m$-independent prefactors) $W_{m}=\overline{J_{m}^{2}\left(\xi r^{\prime}\right)}$. Thus the energy per mode deposited in the $m^{\text {th }}$ subspace is

$$
\varepsilon_{m}=\overline{J_{m}^{2}\left(\xi r^{\prime}\right)} / n_{m}
$$

We conclude

$$
U\left(r, r^{\prime}\right) \sim \overline{J_{o}^{2}\left(\xi r^{\prime}\right) J_{o}^{2}(\xi r)} / n_{o}+2 \sum_{m>0} \overline{J_{m}^{2}\left(\xi r^{\prime}\right)} \overline{J_{m}^{2}(\xi r)} / n_{m}
$$

These quantities are evaluated numerically and plotted in Figure $6 . \mathrm{W}$ is plotted versus $\mathrm{m}$ for the case of a source at $\omega r^{\prime} / c_{s}=77$ (corresponding to $r^{\prime}=76 \mathrm{~mm}$ at $500 \mathrm{kHz}) \cdot \varepsilon_{\mathrm{m}}$ is obtained by dividing $\mathrm{W}_{m}$ by $n_{m}$, for which we must know R, so we 


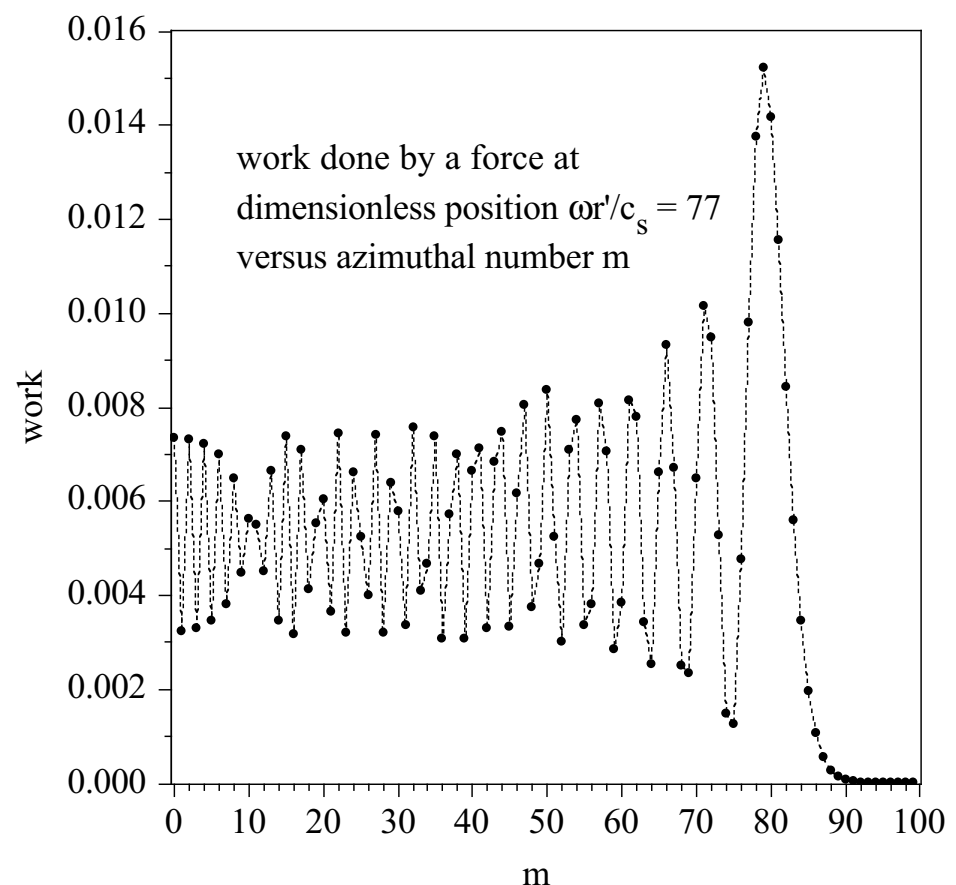

(a)

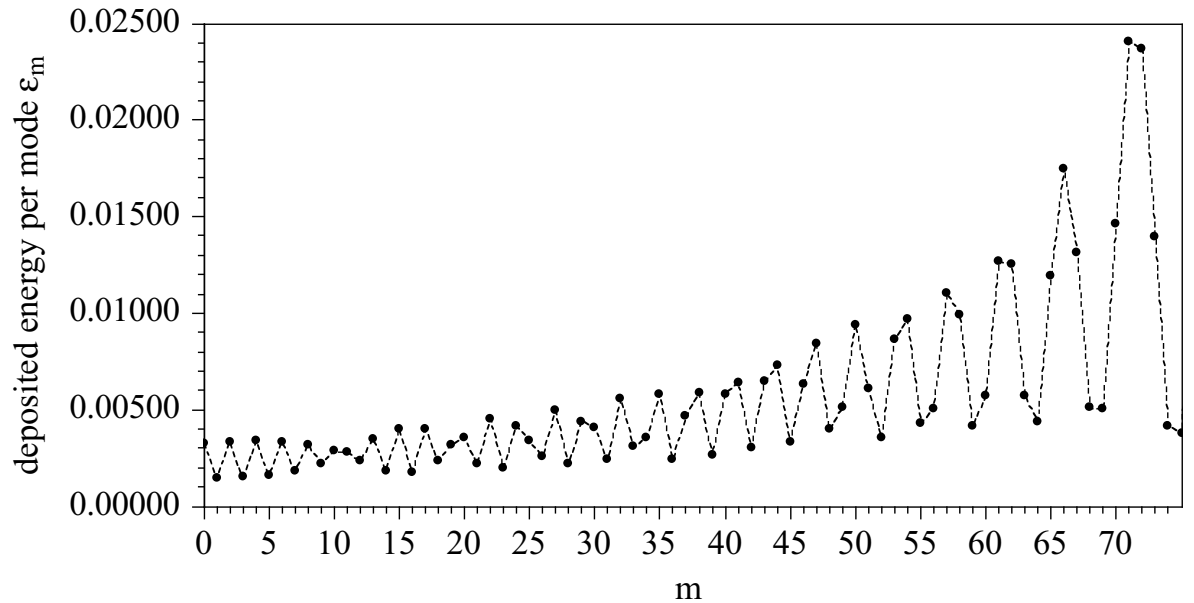

(b)

Fig. 6. a) Work done $\mathrm{W}_{m}=\overline{J_{m}\left(\xi r^{\prime}\right)^{2}}$ in different angular momentum subspaces, for the specified position corresponding to $r^{\prime}=76 \mathrm{~mm}$ from the center if $f=500 \mathrm{kHz}$. b) The energy per mode $\varepsilon_{m}=\mathrm{W}_{m} / n_{m}$ after a source acts at $\omega r^{\prime} / c s=77$. These $\varepsilon g$ are obtained by dividing the $\mathrm{W}_{m}$ of Figure $6 \mathrm{a}$ by $n_{m}=2 / c_{S}^{2}\left(1-|m| c_{s} / \omega r\right) \Theta\left(1-|m| c_{s} / \omega \mathrm{R}\right)+\left(1 / c_{p}^{2}\right)(1-|m|$ $\left.c_{p} / \omega R\right) \Theta\left(1-|m| c_{P} / \omega R\right)$ with $c_{p}=2 c_{s}$. We choose $\omega r / c_{s}=89$, which corresponds to $r=88 \mathrm{~mm}$ if $f=500 \mathrm{kHz}$. The $n$ include the $\mathrm{SH}$ waves, though for short times and low $m$, one might argue that they should not have; to do so would have nearly doubled the $\varepsilon$ at small $m$. The $\varepsilon$ amongst the $\mathrm{m}<50$ differ by factors of no more than four, making them moderately close to equipartition. c) $\mathrm{U}\left(r, r^{\prime}\right)$ (i.e., Eq. 39 using the $\varepsilon$ of Fig. 6 b) for a source at dimensionless distance $\omega r^{\prime} / c_{s}=77$, in a cylinder of dimensionless size $\omega R / c_{s}=89$, versus dimensionless distance $\omega r / c_{s}$. U shows some undulations that will wash out on averaging over a finite frequency band. This shows that energy density over the inner regions after a source acts at finite radius $r^{\prime}$ is nearly constant. 


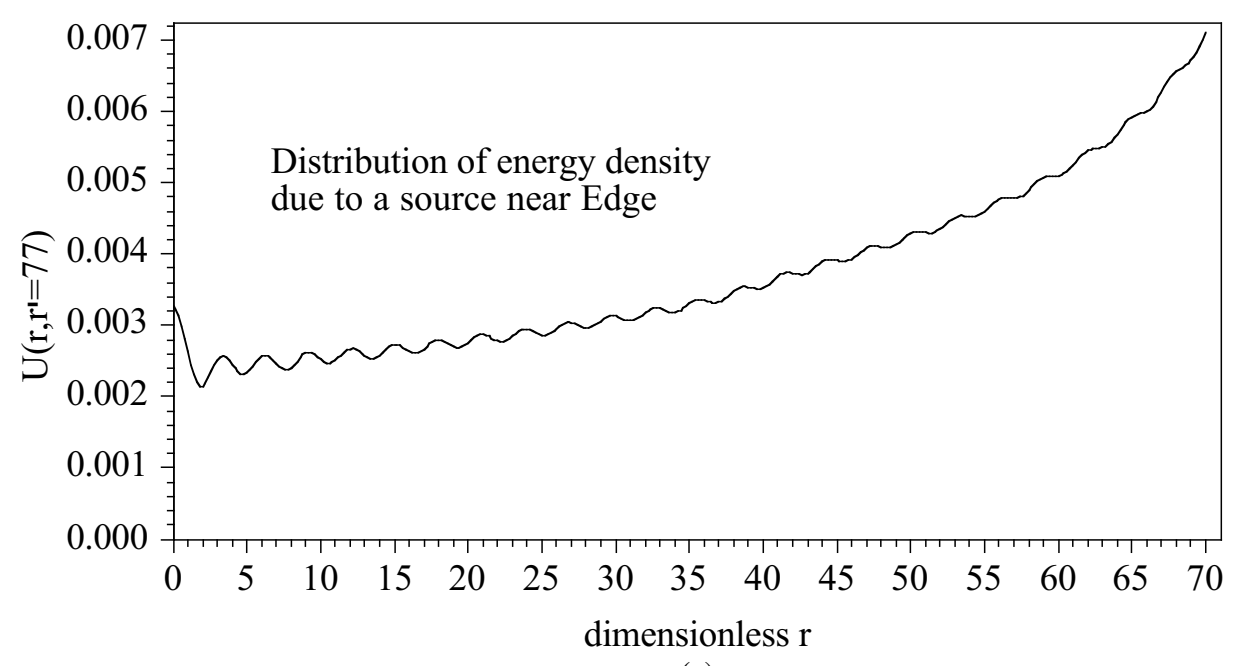

(c)

Fig. 6. Continued.

take $\omega R / c_{\mathrm{s}}=89$ (corresponding to $R=88 \mathrm{~mm}$ if $f=500 \mathrm{kHz}$ ). $U$ is then evaluated and plotted for these parameters versus dimensionless distance $r \omega / c_{s}$ over a region interior to the source, $r<r^{\prime}$. $U$ represents the early time activity of the diffuse field at $r$ due to the source at $r^{\prime}$, before any scattering has changed the values of the $\varepsilon$. Of particular note is that $U\left(r, r^{\prime}\right)$ is approximately independent of $r$ over a range 0 to $2 r^{\prime} / 3$; there is no striking enhancement of energy $U$ at the origin, as there is when the source is at the center. Sources at large distance $r^{\prime}$ generate a field $U$ that, even at times before scattering redistributes the $\varepsilon_{\mathrm{m}}$, is nearly uniform over the inner regions.

\subsubsection{The effect of a poorly sited central transducer.}

If the $\mathrm{C}$ transducer that is nominally at $r^{\prime}=0$ is actually at $r^{\prime}=a \ll$ wavelength, the initial $U(r)$ profile (Eq. 39) due to a source at C is no longer the simple $\overline{J_{o}^{2}(\xi r)}$ calculated above. At large $r$ (and for $a$ small enough that the series (39) converges in a few terms), we may approximate

$$
\overline{J_{m}^{2}(\xi r)} / n_{m} \approx \overline{1 / \pi \xi r} / n_{0}=1 / \pi \xi^{*} r n_{0} .
$$

Therefore

$$
U(a, a) / U(r, a)=\left[{\overline{J_{o}^{2}(\xi a)}}^{2}+2 \Sigma_{m>0}{\overline{J_{m}^{2}(\xi a)}}^{2}\right] \pi \xi^{*} r
$$

The initial ratio $U($ source $) / U(r)$ that had been predicted (Eq. (37)) to be $\pi \xi^{*} r$ is diminished by the first factor above in square brackets that captures the effect of an imperfectly centered transducer C. Figure 7 shows a plot of that factor as evaluated by numerical integration for the case Poisson ratio $=1 / 3$. The diminishment is by a factor of $\sim 2.22$ at $a=c_{\mathrm{S}} / \omega=0.49 \mathrm{~mm} /\left(f_{/ \mathrm{MHz}}\right)$. A half $\mathrm{mm}$ misplacement would at $f=1 \mathrm{MHz}$ - diminish the enhancement by more than a factor of two. The effect is weaker at lower frequencies. It seems that predicted dramatic ratio (37) would be obscured by minor mis-positioning, but it is also apparent that miscentering would have far too weak an effect to be responsible for the discrepancy noted above. 


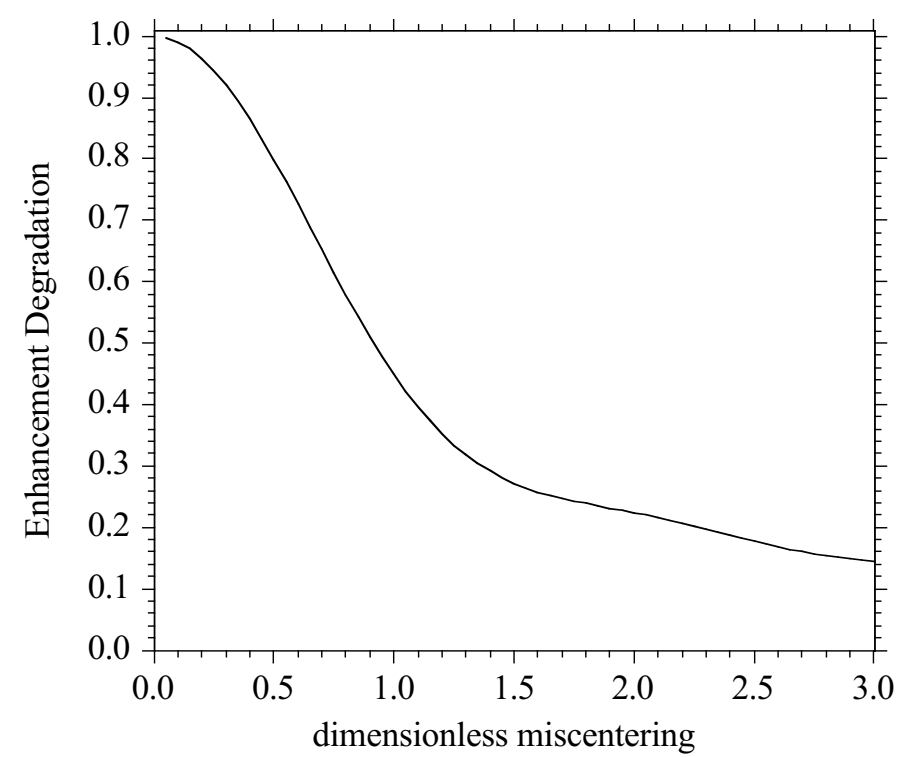

Fig. 7. The factor in equation (41) in square brackets, the factor by which enhancement $\pi \xi \mathrm{q}^{*} r$ of CC over CM is predicted to be diminished by imperfect centering of the transducer at $\mathrm{C}$. It is plotted versus dimensionless miscentering $\omega a / c s$.

\subsubsection{Energy transport between subspaces}

Given a model for how the $\varepsilon_{m}$ evolve from initial conditions, we could use (30) to predict how the mean square fields $U$ and their ratios change in time. The present subsection offers a specific model for that evolution. The model is, however, highly ideal and not meant to be taken as general. Nor is it needed for Section 5's comparisons with measurements. This subsection may therefore be skipped without loss of continuity. It is offered merely for completeness.

Statistical Energy Analysis SEA [32] is usually formulated for a time-independent steady state of energy distribution amongst substructures under the joint influence of continuous energy deposition, incoherent transport, and dissipation. Time-domain SEA equations for that evolution take the form (where $E_{\mathrm{m}}=n_{\mathrm{m}} \varepsilon_{\mathrm{m}}$ is the energy per frequency in subsystem $m$ )

$$
d E_{m} / d t=n_{m} d \varepsilon_{m} / d t=\sum_{n} D_{m n}\left(\varepsilon_{n}-\varepsilon_{m}\right) .
$$

The positive quantities $D$ are related to SEA's "coupling loss factors." They govern the rate at which energy leaks between subspaces. These equations may be conceptualized as analogous to thermal conduction in which the net rate at which energy leaks into subsystem $m$ from subsystem $\mathrm{n}$ is proportional to the difference in energy per mode $\varepsilon$ (analogous to difference in temperature) between those two subsystems and to a thermal conductance $D_{m n}$. This system of equations has - by inspection - a constant solution in which all $\varepsilon$ are equal, corresponding to equipartition. If $D$ is symmetric, it also has the property that total energy $E_{\text {total }}=\Sigma E_{m}=\Sigma \varepsilon_{m} n_{m}$ is a constant, as demanded by energy conservation. Symmetry of $D$ follows from reciprocity ([32] Eq. 4.1.14).

The above equations may be supplemented with extra terms on the right $\Pi_{m}(\mathrm{t})$ $-\sigma_{m} n_{m} \varepsilon_{m}$ representing input power and linear dissipation. In the present section 
we are assuming that all subspaces experience identical dissipation $\sigma$ and as we will only be examining ratios of energy, the effect drops out. Dissipation affects energy ratios between subspaces only if differences in dissipation in different subspaces are sufficiently large compared to transport rates.

The above equations purport to describe how the energy, originally deposited in different subspaces $m$, migrates between subspaces. The details and rates of such migration are of interest as they are measurable in principle and functions of scattering. In this section we describe the energy flows consequent to certain simple assumptions on the $D$ that may or may not obtain in practice. The model is proposed simply for illustration and is not intended to be definitive. Fortunately the SEA prediction that energy is ultimately distributed uniformly is independent of these assumptions on $D$. Let us take, for lack of much information on a better model for the scattering, $D_{m l}=d n_{\mathrm{l}} n_{\mathrm{m}}$ for some fixed $d$. This implies that the energy flow rate from the $1^{\text {th }}$ to the $\mathrm{m}^{\text {th }}$ subspace is $D_{m l} \varepsilon_{l}=d n_{l} n_{m} \varepsilon_{l}=d E_{l} n_{m}$, which is proportional to the total energy in $l$ times the number of modes $n_{m}$ able to accept it in $m$. The model is excessively simple and arguably incorrect for our cylinder (because modes at very different angular momenta are concentrated at different radii, so will presumably be more weakly coupled than modes with similar angular momenta). But the model has the virtue of simplicity and no parameters other than an overall transport time scale $1 / d$; it also has the virtue of permitting an analytic solution.

We rewrite the above as

$$
\begin{aligned}
& \partial_{t}\left\{\begin{array}{l}
\varepsilon_{o}^{P / S V} \\
\varepsilon_{1}^{P / S V / S H} \\
\varepsilon_{2}^{P / S V / S H} \\
\cdot \\
\cdot \\
\varepsilon_{0}^{S H}
\end{array}\right\}=-d\left(n_{0}^{P / S V}+n_{0}^{S H}+2 \sum_{l>0} n_{l}^{P / S V / S H}\right)[I]\left\{\begin{array}{l}
\varepsilon_{o}^{P / S V} \\
\varepsilon_{1}^{P / S V / S H} \\
\varepsilon_{2}^{P / S V / S H} \\
\cdot \\
\cdot \\
\varepsilon_{0}^{S H}
\end{array}\right\} \\
& +d\left[\begin{array}{lll}
n_{0}^{P / S V} & 2 n_{1}^{P / S V / S H} & 2 n_{2}^{P / S V / S H} \ldots ; n_{0}^{S H} \\
n_{0}^{P / S V} & 2 n_{1}^{P / S V / S H} & 2 n_{2}^{P / S V / S H} \ldots ; n_{0}^{S H} \\
\ldots & & \\
n_{0}^{P / S V} & 2 n_{1}^{P / S V / S H} & 2 n_{2}^{P / S V / S H} \ldots ; n_{0}^{S H}
\end{array}\right]\left\{\begin{array}{l}
\varepsilon_{o}^{P / S V} \\
\varepsilon_{1}^{P / S V / S H} \\
\varepsilon_{2}^{P / S V / S H} \\
\cdot \\
\cdot \\
\varepsilon_{0}^{S H}
\end{array}\right\} .
\end{aligned}
$$

Here $\varepsilon_{0}^{(P / S V)}$ is the energy per mode at $m=0$ in $P / S V / R$ waves. $\varepsilon_{m \neq 0}^{(P / S V / S H)}$ are the energies per mode at $m>0$ and include the $S H$ waves under the presumption that $m \neq 0 P / S V / R$ and $S H$ waves of the same $m$ mix rapidly (on a transit time scale). $\varepsilon_{0}^{S H}$ is the energy per mode in $m=0 S H$ waves (presumed to couple only slowly to other modes and so given their own subspace). The $n$ are the corresponding modal densities. The subspaces associated with positive $m>0$ and negative $m<0$ angular momenta have been combined, hence the factors of 2. From Section 4.1, we recall the leading asymptotic approximations

$$
n_{0}^{S H}=\left(L R / 2 c_{s}^{2} \pi\right) \omega ; \quad n_{0}^{P / S V}=\left(L R / 2 c_{p}^{2} \pi+L R / 2 c_{s}^{2} \pi\right) \omega .
$$


The sum over all $n$ is

$$
n_{\text {total }}=n_{0}^{S H}+n_{0}^{P / S V}+2 \sum_{l>0} n_{l}^{P / S V / S H}=\frac{L R^{2} \omega^{2}}{2 \pi}\left[\frac{2}{c_{s}^{3}}+\frac{1}{c_{p}^{3}}\right]
$$

the total modal density. The factor multiplying the Identity matrix in equation (43) may be seen to be $d$ times the total modal density.

We desire a solution that can match initial conditions $\varepsilon_{0}^{S H}=0 ; \varepsilon_{m \neq 0}^{P / S V / S H}=0$; $\varepsilon_{0}^{P / S V} \neq 0$. The solution must be composed of the following two eigenstates of the SEA dynamics ( with $\mathrm{y}=\left(n_{\text {total }}-n_{0}^{P / S V}\right) / n_{0}^{P / S V}=\left(2 R \omega / c_{s}\right)\left(1+K^{3} / 2\right) /\left(1+K^{2}\right)$ $-1=\left(17 R \omega / 10 c_{s}\right)-1$ where $K$ is the ratio of wave speeds $c_{s} / \mathrm{c}_{p} \sim$ taken here to be $\frac{1}{2}$, and $\left.\lambda=\mathrm{d} n_{\text {total }}\right)$ For the system of Section $2 \mathrm{y}=303(f / \mathrm{MHz})$. That solution is

$$
\{\varepsilon\}=\left\{\begin{array}{l}
1 \\
1 \\
\cdot \\
\cdot \\
1
\end{array}\right\}+\left\{\begin{array}{l}
y \\
-1 \\
\cdot \\
\cdot \\
-1
\end{array}\right\} \exp (-\lambda t)
$$

The first term is recognized as the equipartitioned late time steady state. The second term is recognized as having zero total energy, a requirement for any time-varying eigenstate of an energy-conserving SEA dynamics.

From this solution we recover $\varepsilon_{0}^{P / S V}=1+y \exp (-\lambda t)$ and $\varepsilon_{1}^{P / S V / S H}=(1-$ $\exp (-\lambda t))$. Therefore, using (37)

$$
\begin{aligned}
U_{r} / U_{\text {center }} & =\frac{\varepsilon_{1}^{P / S V / S H}}{\varepsilon_{0}^{P / S V}}(1-\overline{1 / \xi r \pi})+\overline{1 / \xi r \pi} \\
& =\frac{1-\exp (-\lambda t)}{1+y \exp (-\lambda t)}(1-\overline{1 / \xi r \pi})+\overline{1 / \xi r \pi} .
\end{aligned}
$$

This makes the anticipated transition from the small quantity $1 / \pi \xi^{*} r$ of order 0.01 in our cylinder at time zero, to unity, i.e., equipartition, at late time $t>\ln (y) / \lambda$.

\subsection{Residual coherence}

The above picture for the distribution of energy in diffuse wave fields must be modified to correct two oversights common to theories like SEA that assume incoherence. Enhanced backscatter (EBS - sometimes called weak Anderson localization, and sometimes called elastic enhancement) describes how signal strength backscattered to a source is stronger than otherwise predicted, by factors of two or three $[14,18,19,43,56]$. Dynamical Anderson localization describes how energy transport between subspaces, whether associated with different angular momenta [57-59] or other kinds of subspaces $[17,60]$, must cease at times after the so-called Heisenberg time, regardless of whether or not equipartition has been achieved.

EBS is widely discussed. In time-reversal invariant systems, multiply scattered rays from a source back to the source are phase coherent with rays that travel by the reverse route. They therefore superpose coherently and interfere constructively. Thus the expected energy level at the source is twice the energy level at nearby positions. The effect appears almost immediately - after a few mean free scattering times (a few hundred $\mu$ sec in the present experiments) and diminishes rapidly with distance from 
the source - on a scale comparable to wavelength. On longer time scales enhancement grows beyond a factor of two: after times such that the modes are resolvable (the Heisenberg time $T_{H}=d N / d f=2 \pi n$ ) enhancement grows to a factor of three, still decaying with distance from the source. These EBS factors have been observed both numerically and in ultrasonic experiments $[14,18,19]$.

Anderson localization is distinct from EBS and more subtle. See Lagendijk et al. [61] for a recent general review of localization for a variety of wave types, including electronic and optical. Ultrasound has provided clear laboratory demonstrations of localization [20,21]. In a statistically homogeneous multiply scattering random medium, one may define a Boltzmann diffusivity proportional to mean free path and wave speed. This diffusivity will govern the spread of wave energy for short times and distances. When diffusion is sufficiently slow, however, wave interference can cause energy to be incapable of diffusing over large distances; transport ceases after finite time. Eigenmodes are localized in space. Less well studied is dynamical Anderson localization [57-59], in which wave energy fails to diffuse, not in space, but rather in a dynamical coordinate like angular momentum. The best known example is perhaps the kicked rotor [59] that classically can diffuse in angular momentum but that when quantized can localize. A nearly axisymmetric wave system whose rays scatter between a finite number of neighboring angular momentum states $m$ is related; it has a nominal diffusivity in angular momentum that governs transport at short times, and a failure to diffuse over long ranges $[57,58]$. One consequence is an absence of long range transport in $m$; equivalently, the normal modes of the structure are all localized over short ranges in $m$.

The present circumstances are somewhat different from the standard picture of dynamical Anderson localization of waves in angular momentum $[57,58]$. Our drilled hole scatters waves between very different angular momentum states. All subspaces $m$ are therefore directly coupled to each other by the scattering. This is in contrast to a picture in which each scattering event changes a ray's angular momentum by only small amounts. Nevertheless, the essential physics still applies: transport must cease after some effective Heisenberg time, regardless of whether or not equipartition has been achieved by then. Energy originally deposited by the source into the $m=0$ subspace leaks out to other $m$, at least initially, at a rate $\lambda$ that we can observe in plots such as those of Figure 4 and ascribe to scattering by the hole. But if that rate is too slow, then transport must cease before equipartition is achieved amongst the $\varepsilon_{\mathrm{m}}$, and uniformity of $U(r)$ is not obtained, even at late times.

In an attempt to estimate the strength of this effect and determine whether or not the observed $\lambda$ in figures like 4 are slow enough, we construct the following argument. Consider a set of $\mathrm{N} \gg 1$ subspaces, each of the same modal density $n$ such that total modal density is $\mathrm{Nn}$, with a transient source acting in one of the subspaces, termed the "home" subspace. Consider the case in which coupling leads to an (initial) exponential leakage of energy $\lambda$ out of the home subspace. In the limit of no coupling, $\lambda=0$, the modes of the home subspace are modes of the whole structure. As the coupling is turned on, each mode (of frequency $\varpi$ ) of the home is mixed with a number of order $\lambda n(N-1)$ modes of the rest of the structure, those with frequencies within $\lambda$ of $\varpi$. Thus energy originally deposited in a mode of the home subspace is ultimately distributed amongst $1+\lambda n(N-1)$ modes, only one of which is in the home system. (This argument fails for $\lambda n>1$ where it does not account for mixing back into other modes of the home system.) The steady state late time energy left in the home system is therefore diminished from the original deposition by a factor of $1 /(1+\lambda n(N-1))$ corresponding to partition of the original energy over these $1+\lambda n(N-1)$ modes. This may be compared to the fraction left if it were to achieve full equipartition: $1 / N$. The ratio is $N /(1+\lambda n(N-1))$. For large $\lambda N n$ we therefore expect the late time energy in the home system to exceed equipartition by a factor of 
order $1 / \lambda n$. The effect is significant if $\lambda n$ is small. If the leaking rate $\lambda$ out of the home subsystem is slow compared to the inverse of modal density $n$ in the home system, equipartition is not achieved. As described above our cylinder has $n_{0}^{P / S V / R}=1.145$ $\operatorname{msec}(f / \mathrm{MHz})$, so $\operatorname{lnCC}$ in Figure 4 with $f=430 \mathrm{kHz}$ and an initial slope $\lambda$ of about $0.20 / \mathrm{msec}$ is indeed slow on this scale $(\lambda n=0.07)$; dynamical Anderson localization is very plausible.

In order to get a more precise estimate we turn to numerical simulations and a rudimentary theory [62]. A thorough study of localization amongst multi-coupled substructures is beyond the present scope, but we have examined transport and localization for the case of $N=100$ statistically equivalent GOE random matrix $[30,63]$ substructures, each with unit variance in its off-diagonal elements, each with 100 internal degrees of freedom and identical modal densities $n$, all coupled weakly. A range of coupling strengths is examined, resulting in a set of different values for the leaking rate $\lambda$ and for the key parameter $\lambda n$. Figure 8 shows the factor $Z$ by which energy at late times in the home substructure was found to exceed equipartition at times long after an initial deposition of energy in the home substructure. It also plots the prediction [62], $Z=N\{\sqrt{1+4 / N \lambda n}-1+2 / N\} /\{\sqrt{1+4 / N \lambda n}+1\}$. That $Z$ is greater than unity is an indication of dynamical localization. It is apparent that the cylinder of Sections 2 and 3, with values of $\lambda$ times modal density comparable to those in Figure 8, should be expected to Anderson localize.

\section{Analysis of measured energy profiles}

We now revisit the measurements introduced in Section 3, and analyze them using the concepts developed above. We discuss $P S D$ distribution and evolution in three cylinders: the cylinder presented in Sections 2 and 3, another with no machined holes and thus better axisymmetry, and finally a more heavily machined version of the first cylinder with diminished axisymmetry.

\subsection{Original cylinder}

Figures 4 and 9 show the evolution of the Power Spectral Densities in each of eight frequency bins, for each of four signals, in the cylinder of Figure 1. In Figures 9, the noise has been subtracted before taking the logarithm, and the negative times suppressed. The data sets marked CC and CM correspond to a source at $C$, and received at $C$ with the reed relay and received simultaneously at M $50 \mathrm{~mm}$ from the center. Data sets marked $E C$ and $E M$ correspond to the signals received (again simultaneously) at $C$ and $M$ respectively due to a source at point $E 78 \mathrm{~mm}$ from the center. Key measures on these profiles are summarized in Table 1.

The first three rows of Table 1 after $f_{\text {central }}$ are obtained from theory; $n_{\mathrm{o}}$ is the modal density in the $m=0 \mathrm{P} / \mathrm{SV} / \mathrm{R}$ subspace not including the $m=0 \mathrm{SH}$ waves; $n_{\mathrm{o}}=1.145 \mathrm{msec}(\mathrm{f} / \mathrm{MHz})$ (this should be halved if the interest is in the $m=0$ modes of definite up/down parity) $n_{\text {total }}$ is the total modal density at that frequency $347(f / \mathrm{MHz})^{2}$ msec. $\pi \xi^{*} r=247(f / \mathrm{MHz})$ is predicted incoherent zero-time enhancement, equation (37), of mean square signal at the center over that at a distance $r=50 \mathrm{~mm}$, neglecting EBS.

The next several rows are taken in whole or part from measurements; $\sigma$ is the observed late time slopes of all four profiles, a measure of internal friction. We may define the quality factor of the structure as $Q=\omega / \sigma=6280(f / \mathrm{MHz}) /\left(\sigma / \mathrm{msec}^{-1}\right)$. Typical values are $Q=10^{5}$. We have found that if insufficient attention is paid to minimizing temperature drift during the 1000 repetitions, then the slopes increase at 


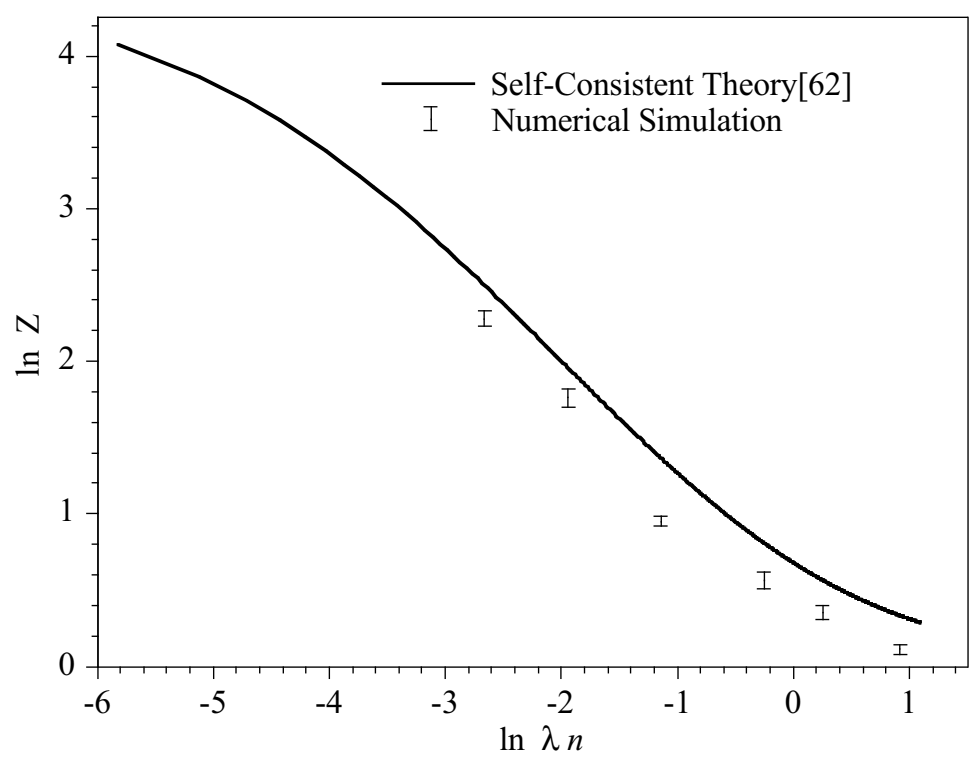

Fig. 8. Results from a numerical study of dynamical localization in a 10000 degree of freedom system composed of $N=100$ nearly uncoupled substructures, each of 100 internal degrees of freedom. $Z$, which depends chiefly on the product of early time energy leaking rate $\lambda$ and local modal density $n$, is the factor by which energy in the home substructure exceeds at late times that which would be predicted by equipartition. Error bars are based on observed fluctuations across numerical ensembles and are equal to the standard deviation amongst 12 samples, divided by $\sqrt{ } 12$. Also plotted (continuous line) are the predictions of a theory [62]: $Z^{\mathrm{SC}}=N\{\sqrt{1+4 / N \lambda n}-1+2 / N\} /\{\sqrt{1+4 / N \lambda n}+1\} \cdot \ln Z^{\mathrm{SC}}$ asymptotes at $\ln (N)$ on the left and $\ln (1)$ on the right. The predictions are qualitatively in accord with the numerical simuations but tend to overestimate the excess. In Section 5, where we observe values of $\ln \lambda n$ between -1 and -4 , we use the theoretical $Z$, but reduce it by $20 \%$.

Table 1. Summary of measurements on the first cylinder. Numbers are taken from the plots of Figures 4 and 9 .

\begin{tabular}{|c|c|c|c|c|c|c|c|c|}
\hline$f_{\text {central }}(\mathrm{kHz})$ & 350 & 429 & 507 & 585 & 664 & 742 & 820 & 898 \\
\hline$n_{\mathrm{o}}(\mathrm{msec})$ & 0.40 & 0.49 & 0.58 & 0.67 & 0.76 & 0.85 & 0.94 & 1.03 \\
\hline$n_{\text {total }}(\mathrm{msec})$ & 42.5 & 63.9 & 89.2 & 119 & 153 & 191 & 233 & 280 \\
\hline$\pi \xi^{*} r$ & 86 & 106 & 125 & 144 & 164 & 183 & 202 & 222 \\
\hline$\lambda\left(\mathrm{msec}^{-1}\right)$ & 0.17 & 0.18 & 0.25 & 0.23 & 0.31 & 0.30 & 0.36 & 0.40 \\
\hline$\sigma\left(\mathrm{msec}^{-1}\right)$ & 0.025 & 0.025 & 0.027 & 0.033 & 0.035 & 0.037 & 0.035 & 0.04 \\
\hline$\lambda^{\mathrm{corr}}\left(\mathrm{msec}^{-1}\right)$ & 0.10 & 0.095 & 0.15 & 0.11 & 0.18 & 0.22 & 0.20 & 0.23 \\
\hline $\ln \left(\mathrm{CC}_{o} / \mathrm{CM}_{\mathrm{o}}\right)$ & -0.25 & 0.60 & 1.7 & 1.30 & -0.50 & 0.70 & 3.0 & 5.0 \\
\hline $\ln \left(\mathrm{CC}_{\infty} / \mathrm{CM}_{\infty}\right)$ & -2.3 & -1.8 & -1.5 & -2.5 & -4.0 & -2.7 & -0.9 & 0.5 \\
\hline $\ln \left(\mathrm{EC}_{\infty} / \mathrm{EM}_{\infty}\right)$ & -5.8 & -4.9 & -4.1 & -4.9 & -6.4 & -5.2 & -2.7 & -1.4 \\
\hline ETE & 257 & 245 & 330 & 493 & 365 & 365 & 299 & 602 \\
\hline $\mathrm{ETE} / \pi \xi^{*} r$ & 2.99 & 2.31 & 2.64 & 4.14 & 2.23 & 1.99 & 1.48 & 2.71 \\
\hline LTEobserved & 3.5 & 3.1 & 2.6 & 2.4 & 2.4 & 2.5 & 1.8 & 1.9 \\
\hline LTE theory & 4.0 & 3.9 & 3.5 & 3.7 & 3.2 & 3.0 & 3.0 & 2.8 \\
\hline$Z^{\text {apparent }}$ & 11.1 & 9.6 & 5.1 & 3.22 & 4.95 & 6.1 & 4.1 & 2.5 \\
\hline
\end{tabular}



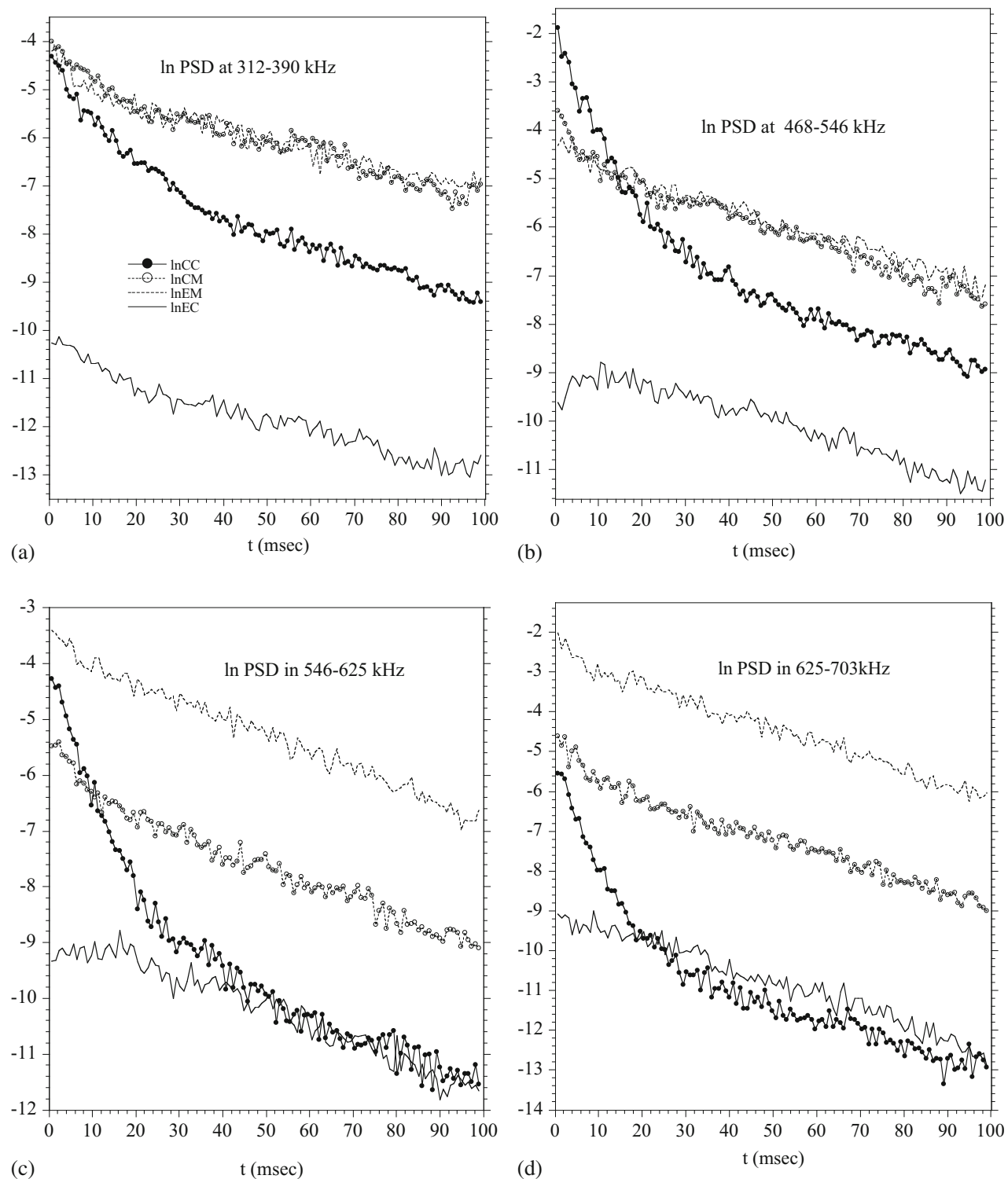

Fig. 9. The power spectral densities in the cylinder of Figure 1 (and plotted for one band in Fig. 4) are plotted here versus time for the other frequency bands.

late time (note how Eq. (1) diminishes at first like the square of time) and the slope at $100 \mathrm{msec}$ becomes an overestimate of average internal friction. Figures 4, 9, 10 and 11 show no sign of this effect.

$\lambda_{o}$ is the observed initial $t=0$ slope of the profile $\ln \mathrm{CC}$ and is a measure (after corrections; see below) of apparent scattering rate out of the $m=0$ subspace. This slope is evaluated using data from times after the theoretical local Heisenberg time $2 \pi n_{o}$, and before the time at which $\ln C \mathrm{C}$ noticeably deviates from linearity, typically $10 \mathrm{msec}$. The early time slope of $\ln \mathrm{CC}$ quantifies the rate at which energy in $m=0$ is diminishing. This diminishment is due to distinct mechanisms. 

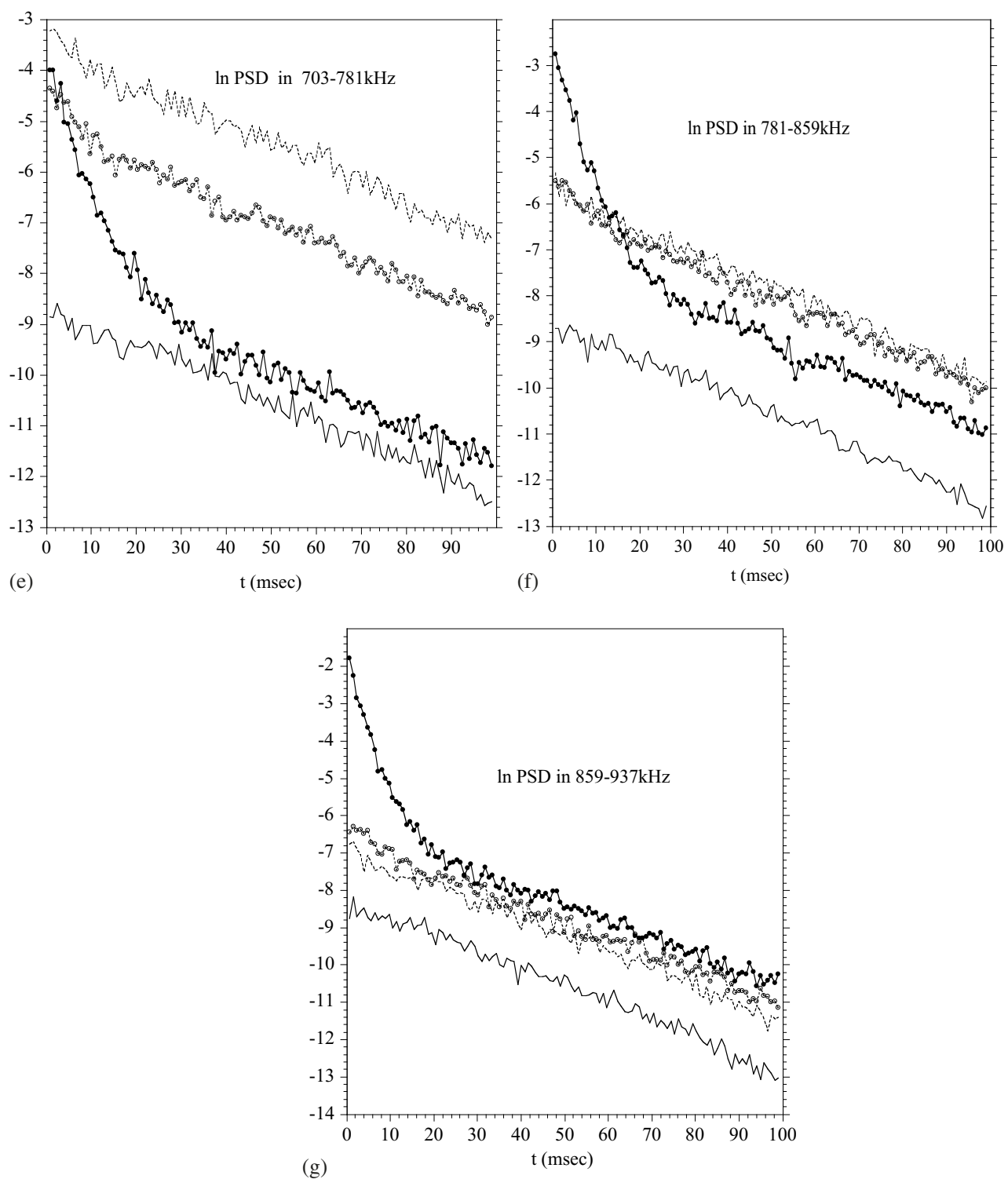

Fig. 9. Continued.

It includes a contribution from transport, as energy is scattered to other angular momenta. It includes a contribution from bulk absorption $\sigma$. It furthermore includes a contribution from loss due to the intrusive dissipation of the $\mathrm{C}$ transducer. That transducer dissipates only the $m=0$ subspace; it concentrates its otherwise small effects $\left(p=0.6 \mathrm{~cm}^{3} / \mathrm{msec}\right.$; see Sect. 2.5$)$ there. We calculate its contribution to losses in the $m=0$ subspace as $p J_{o}(0)^{2} /\left[2 \pi L \int r d r J_{o}(\xi r)^{2}\right]=p \xi^{*} / 2 L R=0.05(f / \mathrm{MHz}) \mathrm{msec}^{-1}$. The loss into the transducer is further augmented by an EBS factor of two or three whenever the transducer is at the source of the field. We conclude that the tabulated values of $\lambda_{o}$ are overestimates of transport rates into other angular momentum subspaces. The table therefore provides a $\lambda$ corrected by subtracting $\sigma$, 

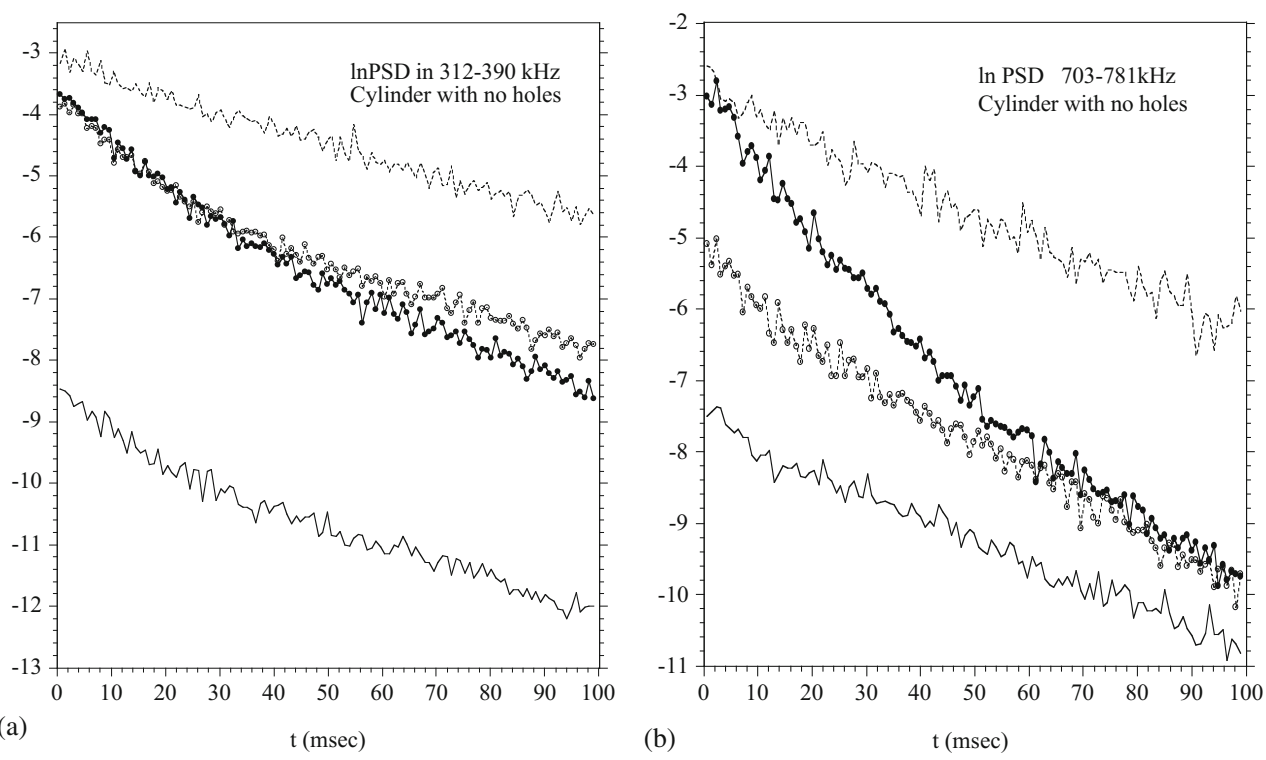

Fig. 10. The power spectral densities in two of the frequency bands, for a cylinder like that of Figure 1, but with only $76 \mathrm{~mm}$ height and, more importantly, with no hole drilled to break axisymmetry. Legend as in Figures 9.
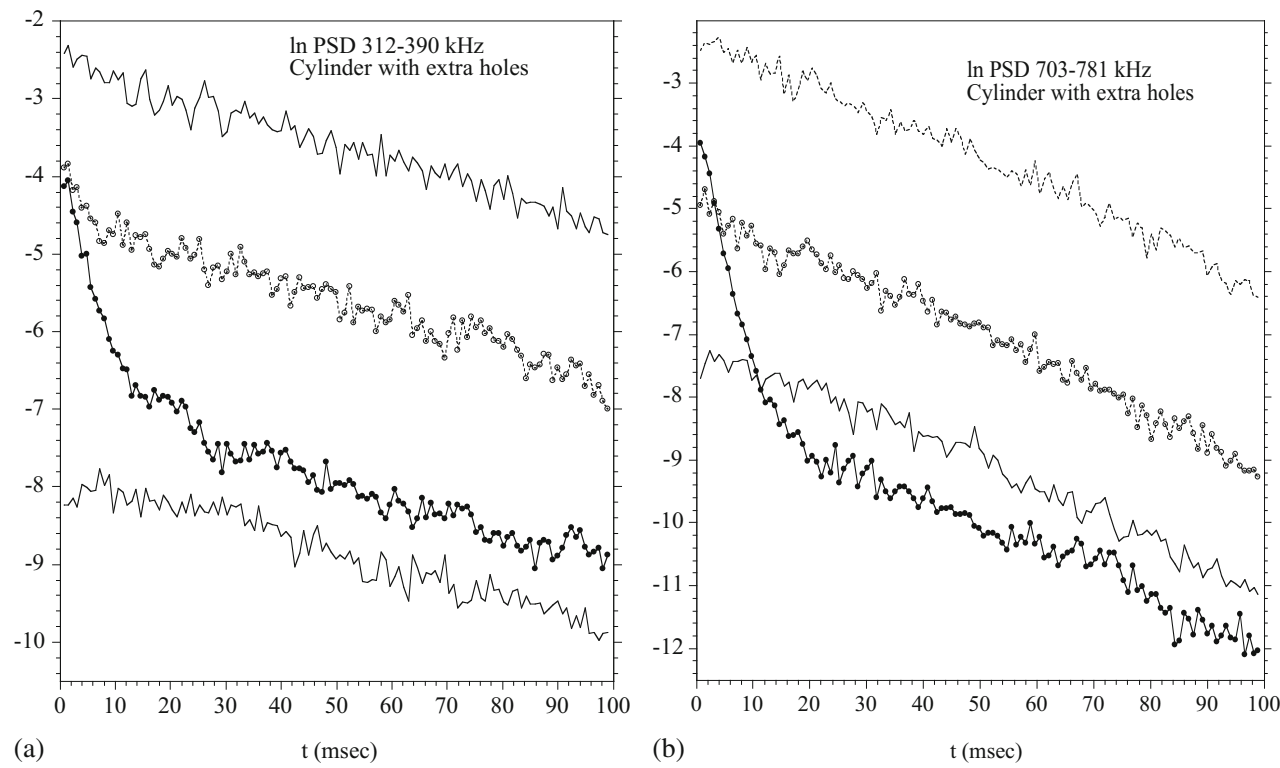

Fig. 11. The power spectral density in each of two frequency bands for a cylinder with more machining, to better break axisymmetry and up/down reflection symmetry. Legend as in Figures 9. 
and subtracting the estimate $0.15(f / \mathrm{MHz}) / \mathrm{msec}$ for the extra losses in the $\mathrm{C}$ transducer: $\lambda^{\text {corr }}=\lambda_{o}-\sigma-0.15(f / \mathrm{MHz})$.

We may attempt a connection between the $\lambda^{\text {corr }}$ and a scattering cross section $\Sigma$ for the hole: $\lambda^{\text {corr }}=c \Sigma / V^{\text {eff }}$, where $V^{\text {eff }}$ is an effective volume, equal to $2 \pi L R^{2}=$ $4800 \mathrm{~cm}^{3}$ (twice the actual volume due to the non uniform distribution of the $m=0$ energy.) A $\lambda^{\text {corr }}$ of the observed order, 0.10 to $0.20 / \mathrm{msec}$, thus corresponds to a cross section of about 1.5 to $3.0 \mathrm{~cm}^{2}$. This cross section is not unreasonable. To the extent that it exceeds what we might have expected for a $12 \mathrm{~mm} \times 4 \mathrm{~mm}$ tapped hole at shear wavelengths of order $5 \mathrm{~mm}$, one could posit the existence of other sources of scattering. Perhaps the nail supports or the transducers provide some scattering. Scattering by polycrystalline microstructure is unlikely to be significant; such scattering is weak in aluminum below $1 \mathrm{MHz}$ unless crystallites are large, and would in any case be expected to be a strong function of frequency [64].

$\ln \left(\mathrm{CC}_{\mathrm{o}} / \mathrm{CM}_{\mathrm{o}}\right)$ represents the relative strengths of these two PSDs at time zero. It is obtained by linear extrapolation of the measured $\ln (\mathrm{CC} / \mathrm{CM})$ to zero time using the data from times at and after the local Heisenberg time $2 \pi n_{\mathrm{o}}$, and before the ratio noticeably deviates from linearity.

$\ln \left(\mathrm{EC}_{\infty} / \mathrm{EM}_{\infty}\right)$ represents the relative strength of these two PSDs at late time. It is obtained by averaging $\ln (\mathrm{EC} / \mathrm{EM})$ over late times, defined as a period during which there is no discernable trend, typically from 30 to $100 \mathrm{msec}$. (Values for $\ln \mathrm{CC}_{\infty} / \mathrm{CM}_{\infty}$ are obtained by similar averaging.) On presuming late time mean square fields $U$ at $\mathrm{C}$ and at $\mathrm{M}$ due to a source at $\mathrm{E}$ are equal, this number quantifies the relative sensitivities of the two transducers at $\mathrm{C}$ and $\mathrm{M}$. The late time equality of $\mathrm{U}$ at $\mathrm{C}$ and $\mathrm{M}$ due to a source at $E$ follows from our knowledge that $\mathrm{E}$ deposits energy roughly uniformly over the lower values of $\mathrm{m}$, and over smaller values of $\mathrm{r}$, as illustrated in Figure 6. It therefore creates an initial field that is close to equipartitioned. After some transport (i.e. at large $t$ ) the field must be even better equipartitioned. Thus we compare the powers EC and EM at late times to assess the relative sensitivities of the sensors at $\mathrm{C}$ and $\mathrm{M}$. That EC/EM rises in time by a factor of about three (consistent with Figure 6's estimate that EC starts below equipartition and so must rise due to transport), and completes its changes within $20 \mathrm{msec}$, is a further indication that transport is well finished by $t=100 \mathrm{msec}$ and that EC/EM has achieved its steady state.

Observed early time enhancement ETE is defined as ETE $=\left(\mathrm{CC}_{\mathrm{o}} / \mathrm{CM}_{\mathrm{o}}\right)$ $\left(\mathrm{EM}_{\infty} / \mathrm{EC}_{\infty}\right)$ and represents the observed enhancement of energy $\mathrm{CC}$ over $\mathrm{CM}$ at early times, as corrected by the calibration provided by EM/EC at late times. This quantity may be compared to the enhancement predicted in Sections 3 and $4: \pi \xi^{*} r$. Values of $\mathrm{ETE} / \pi \xi^{*} r$ are of order three, consistent with predictions of enhanced backscatter. At the higher frequencies, they drop to order two. Thus we corroborate the assertion in Sections 3 and 4.3, that energy densities at time zero in the center will be enhanced over energy densities elsewhere by the large number $\pi \xi^{*} r$, though also enhanced by EBS.

We suggest two possible explanations for our short-time EBS measure (i.e. $\mathrm{ETE} / \pi \xi^{*} r$ ) to be closer to 2 than 3 at higher frequencies. It may be that there is some miscentering as illustrated in Figure 7. Perfect centering is difficult and high frequencies magnify the effect of imperfect centering. We estimate after repeated measurements that the center of the $\mathrm{C}$ transducer is placed with an error of no more than $0.5 \mathrm{~mm}$. (Furthermore, the transducer itself has a diameter of $1.6 \mathrm{~mm}$ and the precise center of its sensitivity is unclear.) An error of $0.5 \mathrm{~mm}$ at $1 \mathrm{MHz}$ leads to degradation by a factor of 2.22 , as shown in Figure 7 . This would be sufficient to explain the observed EBS factors. As an alternative explanation one could hypothesize that EBS, though equal to 3 in an isolated subsystem, is lessened by leaking into other systems when that leaking is significant on a scale of the local Heisenberg time [64]. Indeed 
if transport $\lambda$ were fast enough, EBS would start at two but would not approach 3 until $t=2 \pi n_{\text {total }}$. Lacking a theory for EBS in a system of multiple substructures, we cannot tell which explanation is more likely.

Late time enhancement LTE is a measure of residual coherence and failure to achieve equipartition; it is defined by $\mathrm{LTE}=\ln \left(\mathrm{CC}_{\infty} / \mathrm{CM}_{\infty}\right)\left(\mathrm{EM}_{\infty} / \mathrm{EC}_{\infty}\right)$. This number is constructed to be independent of transducer sensitivities and amplifications and thus represents the corresponding ratio of mean square fields $U$ at different positions; it would be zero if energy were to be fully incoherent and equipartitioned, i.e, in the absence of Anderson localization or enhanced backscatter.

If transport were rapid such that there is little Anderson localization, LTE would simply be the ln of the EBS factor (i.e., ln 2 at times well before the total system Heisenberg time $2 \pi n^{\text {total }}$, ln 3 after). In the absence of any transport, such that all energy remains in $m=0$, CC would experience the full EBS factor of 3 (because 50-100 msec is late compared to local Heisenberg time $2 \pi n_{\mathrm{o}}$ ). In that limit LTE would (still taking $\mathrm{EM}_{\infty} / \mathrm{EC}_{\infty}$ to nevertheless calibrate the transducers) be $\ln \left(3 \pi \xi^{*} r\right.$ ).

However, in the parameter regime of these measurements, in which $Z$ is much less than $\pi \xi^{*} \mathrm{r}$, theoretical LTE is approximately LTE theory $^{\text {the }} \ln$ (EBS times $Z$ taken from Figure 8). It is not clear whether the appropriate EBS value is 3 or 2 or something in between, as the effective Heisenberg time is not obvious for this purpose, $2 \pi n_{\mathrm{o}}$ or $2 \pi n^{\text {total }}[65]$. Thus we split the difference and choose the geometric mean $\sqrt{ } 6$; the uncertainty is in any case mild compared to measurement uncertainties. LTE ${ }^{\text {theory }}=$ $\ln \sqrt{ } 6+\ln Z^{S C}\left(\lambda^{\text {corr }} n_{\mathrm{o}} / 2\right)-0.2$, where 0.2 is subtracted to bring self consistent theory $Z^{S C}$ in Figure 8 closer to the numerical simulations shown in Figure 8 . We use $\lambda^{\text {corr }} n_{\mathrm{o}} / 2$ rather than $\lambda n_{o}$ after noting that for these purposes the modal density in the home substructure is $\mathrm{n}_{o} / 2$, not $n_{o}$, due to how the cylinder's nominal up/down reflection symmetry splits each subspace $m$ into nominally uncoupled even and odd parts.

We see a general agreement between the observed and theoretical LTE, thus confirming our hypothesis that this system exhibits dynamical Anderson localization. Energy at late times is stronger at $\mathrm{C}$ than at $\mathrm{M}$ by significant factors, $\exp (\mathrm{LTE})$, as high as 40. Predicted enhancement according to our theories is generally greater (thus localization is stronger) than observed, by factors of order 3 , though the trends are the same. Differences are perhaps ascribable to underestimates of $\lambda^{\text {corr }}$ or extra losses in $\mathrm{CC}$ relative to $\mathrm{CM}$ due to the intrusiveness of the $\mathrm{C}$ transducer, or the lack of exact correspondence between the 100 equally coupled statistically equivalent subsystems of the simulations and theory in Figure 8 and the more than 100 non-equal-sized non-equally coupled subsystems in the cylinder.

Dynamical localization is revealed also, without recourse to intepretations of $\mathrm{EC} / \mathrm{EM}$ as a calibration, by the quantity $Z^{\text {apparent }}$ defined as $\pi \xi^{*} r$ $(\mathrm{CC} / \mathrm{CM})_{\infty} /(\mathrm{CC} / \mathrm{CM})_{0}$. (This is the quantity discussed in IV.C.2 as taking the value 106/12 for the data of Figure 4.) This is the factor by which CC/CM has not achieved equipartition at time 100 (and inasmuch as CC/CM is in a steady state at late times, presumably at $\mathrm{t}=\infty$ also). If EBS is unchanged from time zero to $t=100 \mathrm{msec}$, this is simply $Z$, the factor by which late time energy in $m=0$ exceeds its equipartitioned share. $Z^{\text {apparent }}$ would be unity in the absence of localization.

\subsection{Cylinder without hole}

To provide further evidence for the above picture we have repeated these measurements on a cylinder with better axisymmetry, one for which we therefore expect corrected $\lambda$ to be much less, and correspondingly expect LTE to be much greater. A second cylinder was prepared (178 $\mathrm{mm}$ diameter $\times 77 \mathrm{~mm}$ height) without 
Table 2. Summary of measurements on the cylinder with no holes.

\begin{tabular}{|l|l|l|l|l|l|l|l|l|}
\hline$f_{\text {central }}(\mathrm{kHz})$ & 350 & 429 & 507 & 585 & 664 & 742 & 820 & 898 \\
\hline$n_{\mathrm{o}}(\mathrm{msec})$ & 0.31 & 0.38 & 0.45 & 0.52 & 0.59 & 0.65 & 0.72 & 0.79 \\
\hline$n_{\text {total }}(\mathrm{msec})$ & 32.7 & 49.2 & 68.7 & 91.6 & 118 & 147 & 179 & 216 \\
\hline$\pi \xi^{*} r$ & 86 & 106 & 125 & 144 & 164 & 183 & 202 & 222 \\
\hline & & & & & & & & \\
\hline$\lambda_{0}\left(\mathrm{msec}^{-1}\right)$ & .077 & .15 & .16 & 0.14 & 0.14 & .12 & .10 & .11 \\
\hline$\sigma\left(\mathrm{msec}^{1}\right)$ & .026 & 0.025 & .027 & 0.027 & .030 & .031 & .033 & .034 \\
\hline$\lambda^{\text {corr }}\left(\mathrm{msec}^{-1}\right)$ & -.01 & 0.04 & 0.04 & 0.00 & -.01 & -0.05 & -0.08 & -0.09 \\
\hline $\ln \left(\mathrm{CC} / \mathrm{CM}_{\circ}\right)$ & 0.3 & 1.4 & 2.25 & 1.9 & 1.25 & 2.3 & 3.1 & 4.2 \\
\hline $\ln (\mathrm{CC} / \mathrm{CM})_{100 \mathrm{~ms}}$ & -.8 & -.15 & -.25 & -.75 & -1.5 & .20 & .6 & 1.8 \\
\hline $\ln (\mathrm{EC} / \mathrm{EM})_{100 \mathrm{~ms}}$ & -6.5 & -5.4 & -5.25 & -5.5 & -6.0 & -4.5 & -2.7 & -1.6 \\
\hline & & & & & & & & \\
\hline $\mathrm{ETE}$ & 900 & 900 & 1800 & 1640 & 1800 & 900 & 330 & 330 \\
\hline ETE $/ \pi \xi * \mathrm{r}$ & 10.4 & 8.5 & 14.5 & 11.4 & 11.0 & 4.9 & 1.63 & 1.48 \\
\hline $\mathrm{LTE}$ observed & 5.7 & 5.3 & 5.0 & 4.75 & 4.5 & 4.7 & 3.3 & 3.4 \\
\hline $\mathrm{Z}^{\text {apparent }}$ & 29 & 22.5 & 10.3 & 10.2 & 10.5 & 22.4 & 16.6 & 3.0 \\
\hline
\end{tabular}

a hole drilled in the side. $M$ was again at $50 \mathrm{~mm}$. Sample PSD profiles are shown in Figures 10. Table 2 summarizes the measurements on this cylinder.

Values of $\sigma$ as ascertained from the late time slopes of EM were again about $0.03 / \mathrm{msec}$. Values of $\lambda$ taken from the early time slopes of CC were much less in this cylinder without hole than in the cylinder with hole. $\lambda^{\text {corrected }}$ values (using $\lambda^{\text {corrected }}=\lambda_{0}-\sigma-0.19(\mathrm{f} / \mathrm{MHz})$ as appropriate for the $77 \mathrm{~mm}$ thick cylinder $)$ were negative or within uncertainty of zero. Of course negative transport rates are unphysical. We offer brief speculations as to how corrected $\lambda$ might be negative by noting that, if localization is strong as it clearly is here, then the early times in CC over which $\lambda_{0}$ is evaluated will include times in which localization has slowed the transport. Thus our measurement of $\lambda_{0}$ may underestimate the actual $\lambda_{0}$. Alternatively, miscentering would distribute the intrusiveness of the $\mathrm{C}$ transducer over two or more $m$ subspaces, diluting its effect and rendering our estimate $0.19(\mathrm{f} / \mathrm{MHz})$ too large at high frequencies.

We conclude that transport is much slower in this cylinder than it is in Figures 4 and 9 but recognize that we cannot accurately estimate transport rates. In that this cylinder without hole exhibits no discernable transport, we confirm the supposition that the drilled hole was responsible for the transport seen in the original cylinder.

The profiles $\ln (\mathrm{EM})$ show almost no deviations from linearity in time; decay is nearly purely exponential. This is consistent with a lack of transport; EM neither loses nor gains due to transport, and is dissipated only by bulk losses $\sigma$. The other PSD in Figure 10 are not, however, decaying purely exponentially. This is not inconsistent with a lack of transport, as differential absorption ( $m=0$ being more strongly attenuated due to the intrusiveness $p$ of the transducer $\mathrm{C}$ ) will manifest as decay curvature $[24,66,67]$ in any PSD, especially those measured at $\mathrm{C}$ or having source at $\mathrm{C}$.

That transport is so slow is also evident in that our key ratio $(\mathrm{CC} / \mathrm{CM})$ has not achieved a steady state value, even at $t=100 \mathrm{msec}$ (at which time it is still decaying, at rates between 0.01 and $0.02 / \mathrm{msec}$ ). This may be because transport is so slow that it is still ongoing at $t=100$. But even if there were no transport, this behavior could be explained as due to non-uniformity of dissipation associated with $C$ 's intrusiveness, $m=0$ modes being more strongly dissipated and not replenished by transport from other subspaces; hence the more rapid decay of CC. 
Table 3. Summary of measurements on the cylinder with extra holes and a bevel.

\begin{tabular}{|l|l|l|l|l|l|l|l|l|}
\hline$f_{\text {central }}(\mathrm{kHz})$ & 350 & 429 & 507 & 585 & 664 & 742 & 820 & 898 \\
\hline$n_{\mathrm{o}}(\mathrm{msec})$ & 0.40 & 0.49 & 0.58 & 0.67 & 0.76 & 0.85 & 0.94 & 1.03 \\
\hline$n_{\text {total }}(\mathrm{msec})$ & 42.5 & 63.9 & 89.2 & 119 & 153 & 191 & 233 & 280 \\
\hline$\pi \xi^{*} r$ & 86 & 106 & 125 & 144 & 164 & 183 & 202 & 222 \\
\hline & & & & & & & & \\
\hline$\lambda_{0}\left(\mathrm{msec}^{-1}\right)$ & .28 & .311 & .40 & 0.39 & 0.40 & .41 & .42 & .56 \\
\hline$\sigma\left(\mathrm{msec}^{-1}\right)$ & .022 & 0.029 & .030 & 0.031 & .038 & .047 & .045 & .055 \\
\hline$\lambda^{\text {corr }}\left(\mathrm{msec}^{-1}\right)$ & 0.20 & 0.22 & 0.29 & 0.27 & 0.26 & 0.25 & 0.25 & 0.37 \\
\hline $\ln \left(\mathrm{CC}_{\circ} / \mathrm{CM}_{\circ}\right)$ & 0.0 & .75 & 1.6 & 1.2 & 0.4 & 1.3 & 2.8 & 4.5 \\
\hline $\ln \left(\mathrm{CC}_{\infty} / \mathrm{CM}_{\infty}\right)$ & -2.2 & -2.1 & -1.75 & -2.9 & -3.7 & -2.8 & -1.5 & 0.0 \\
\hline $\ln \left(\mathrm{EC}_{\infty} / \mathrm{EM}_{\infty}\right)$ & -5.3 & -4.35 & -3.8 & -4.6 & -5.8 & -4.8 & -2.9 & -1.4 \\
\hline & & & & & & & & \\
\hline ETE & 200 & 164 & 221 & 330 & 493 & 446 & 299 & 365 \\
\hline ETE $/ \pi \xi * r$ & 2.3 & 1.55 & 1.77 & 2.64 & 3.0 & 2.44 & 1.48 & 1.64 \\
\hline LTEobserved & 3.1 & 2.25 & 2.05 & 1.7 & 2.1 & 2.0 & 1.4 & 1.4 \\
\hline LTE theory & 3.1 & 2.9 & 2.5 & 2.5 & 2.4 & 2.3 & 2.3 & 1.9 \\
\hline $\mathrm{Z}^{\text {apparent }}$ & 9.5 & 6.1 & 4.4 & 2.4 & 2.7 & 3.0 & 2.7 & 2.5 \\
\hline
\end{tabular}

The EM to EC ratios at $t=100 \mathrm{msec}$ are, in this cylinder, unreliable as an indicator of relative sensitivity of transducers. We recall that the ratios EC/EM rose with time in the first cylinder, but here they appear to fall. A falling ratio is not at all consistent with evolution towards equipartition after an initial deposition as described in Figure 6 that favors energy at $M$. It is consistent with excess losses in $m=0$ and no transport. If we nevertheless construct ETE and LTE using EC/EM at $t=100 \mathrm{msec}$ for calibration (see Table 2) we find values of ETE $/ \pi \xi^{*} r$ as high as 14 . This ratio ought be EBS, which in this case of very weak transport ought be 3 . One presumes then that calibration using $\mathrm{t}=100 \mathrm{msec}$ is incorrect by factors up to 4.5 . No estimate can be made of theoretical LTE, as $\lambda^{\text {corr }}$ is poorly known (so theoretical LTE has been omitted from the table), but the observed values are greater than those listed for the first cylinder (Table 1) and so are consistent with greater localization associated with slower transport.

Similarly, in that CC/CM has not achieved its steady state, even at $t=100$, the quoted values of apparent $Z$ are not meaningful.

In sum, we make qualitative assessment that transport is exceptionally weak. But the values of $\lambda^{\text {corrected }}$ and LTE and ETE and $Z^{\text {apparent }}$ quoted in Table 2 are not as robust as they were in Table 1 . The significant qualitative differences between Figures 9 and 10 and between Tables 1 and 2 nevertheless provide striking evidence for the effect of weak axisymmetry breaking on transport and localization.

\subsection{Cylinder with extra scattering}

As an alternate corroboration of the theory we add scatterers to the first cylinder, further breaking the axisymmetry by machining five $3 \mathrm{~mm}$ diameter $3 \mathrm{~mm}$ deep flat bottomed holes on the bottom face. They are placed at points $r=10,25,40,65$ and $80 \mathrm{~mm}$ from the center, and $\theta=36,108,180,252$ and 324 degrees respectively from the $12 \mathrm{~mm}$ hole. We also add an axisymmetric $6 \times 6 \mathrm{~mm} 45$ degree bevel to the bottom edge to break the up/down reflection symmetry. Representative PSD profiles are shown in Figure 11. M was, as previously, at $r=50 \mathrm{~mm}$. $E$ was at $r^{\prime}=78 \mathrm{~mm}$.

Table 3 lists the main features. As anticipated, the extra holes have increased the corrected $\lambda$, by factors between 1.5 and 2. Internal friction $\sigma$ has been 


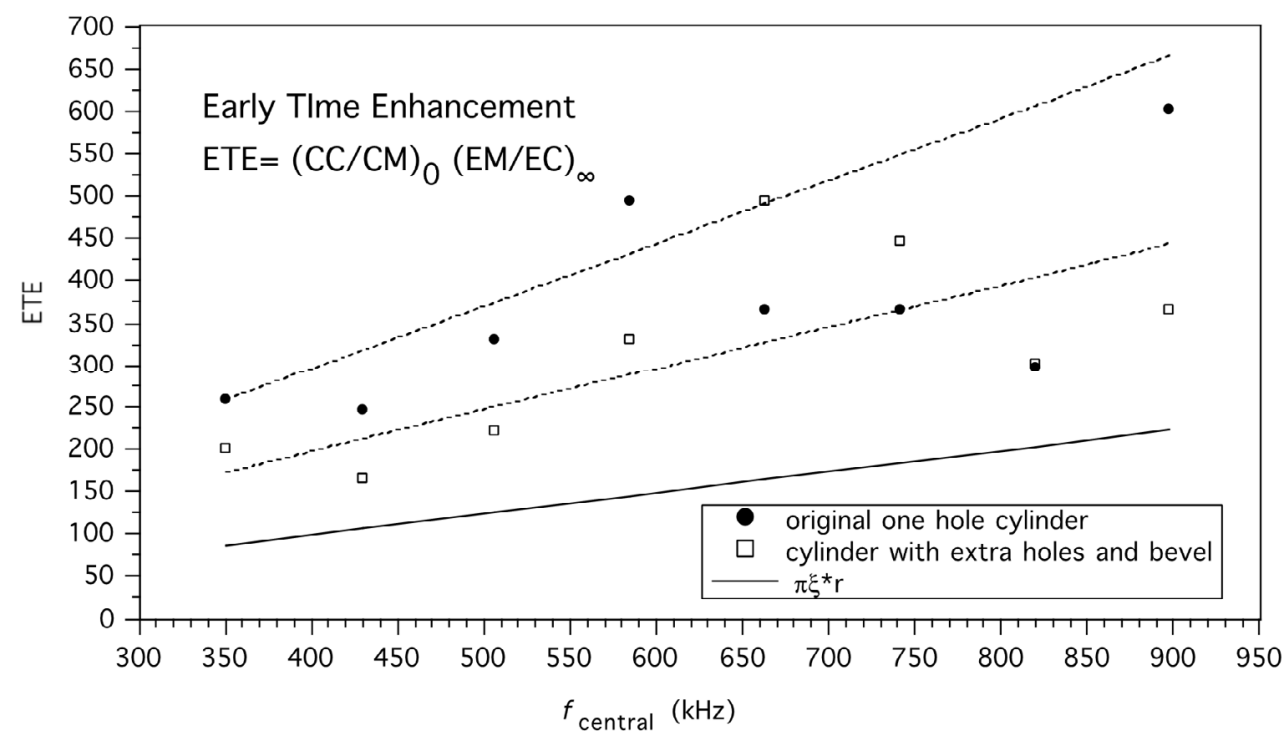

Fig. 12. Comparison of early time enhancement in the original cylinder and after further machining. The two dashed lines are $2 \pi \xi^{*} r$ and $3 \pi \xi^{*} r$. Theory predicts early time enhancement should lie between the two dashed lines. That energy at $\mathrm{C}$ where the source acts is enhanced at early times over that at $M$ is apparent.

increased slightly, consistent with observations (e.g. 20) that machining induces a surface damage that manifests in extra losses. LTE ${ }^{\text {theory }}$ is now calculated as $\ln \sqrt{ } 6+$ $\ln \left(Z^{S C}\left(\lambda^{\text {corr }} n_{o}\right)\right)-0.2$ (we no longer use $n_{\mathrm{o}} / 2$ in this formula because the bevel has broken the up/down reflection symmetry so the home subsystem modal density is now $n_{\mathrm{o}}$ not $n_{\mathrm{o}} / 2$ ). Both measured and theoretical LTE have been substantially diminished by the machining. The observed enhancement, as quantified by the EBS value $\mathrm{ETE} / \pi \xi^{*} r$, is less than three, even more so than in the original cylinder. That this weak EBS occurs at low frequencies suggests that miscentering cannot be responsible for all of it. The conjectured culprit is therefore, as hypothesized for the first cylinder, weakened elastic enhancement in open systems [65].

Figures 12 and 13 summarize the key quantities from these three tables. Figure 12 shows that the early time enhancement of the signal CC over the signal CM, argued in Section 3 to be large, $\pi \xi^{*} r$, is indeed large. Actual enhancements are slightly greater, consistent with EBS. Figure 13 shows that the enhancement at the center, while diminished by transport, continues to late time, in contrast to what would be expected were the field to become equipartitioned. Late time enhancement is observed to be anti-correlated with scattering strength $\lambda^{\text {corr }}$ and modal density $n_{0}$, as expected by models of dynamical localization.

\section{Summary}

We have measured the time-dependent diffuse ultrasonic energy density at points on the flat top surface of a nominally axisymmetric body. The distribution in space is shown theoretically to be a function of the distribution of energy amongst different angular momentum subspaces, so its evolution is a function of diffuse transport of energy between angular momentum subspaces. A striking feature of that timedependent distribution is a large concentration, at early times, of the mean square 


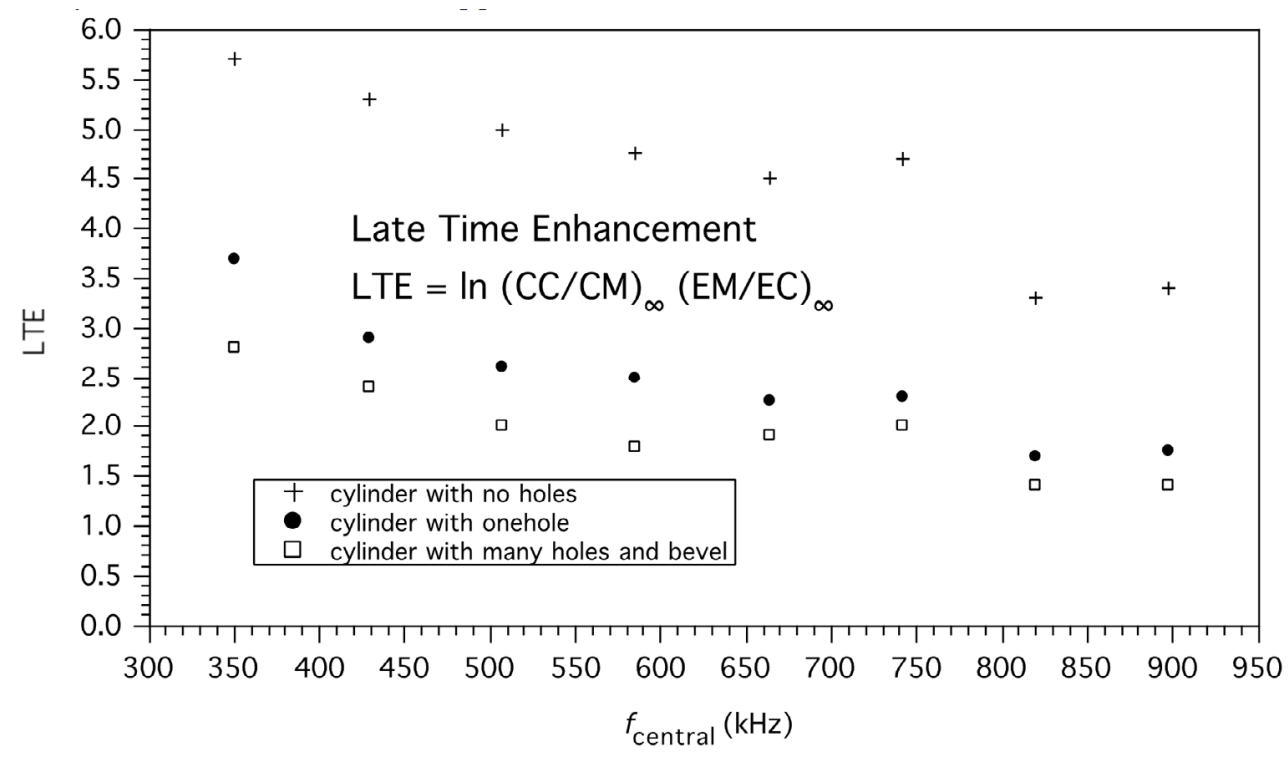

Fig. 13. The late time enhancement of energy at the center $C$ over the energy at point $M$ due to a source acting at the center. If full equipartition were to be achieved this quantity would be zero. Observed enhancements are between $\mathrm{e}^{1.5}$ and $\mathrm{e}^{5.7}$ and are comparable to what is predicted by a combination of EBS and our numerical simulations of dynamical localization. The expected greater enhancement for specimens with better axisymmetry and for lower frequencies (i.e. for smaller values of $\lambda n_{0}$ ) is apparent.

signal at the cylinder center due to a source at that center, followed by a subsequent slow relaxation of that concentration. Measurements show that the initial concentration conforms to these simple incoherent diffuse field theory predictions, as modified by weak Anderson localization (EBS). We further find that the subsequent relaxation does not proceed all the way to the equipartition and uniformity predicted by incoherent diffuse field theory. It is shown that dynamical Anderson localization is likely responsible for this. The observed degree of localization is slightly weaker than predicted by numerical simulations of coupled random matrices but trends as predicted with the inverse of local modal density and scattering rate. Precise comparisons between experiments and theory and numerics are complicated, however, by intrusiveness and losses in the $\mathrm{C}$ transducer. They are also complicated by theoretical uncertainty for the strength of enhanced backscatter in open substructures and by imprecise correspondence between our random matrix model parameters and those of the ultrasonic system.

These ideas are of interest in general for mesoscopic waves; Anderson localization and enhanced backscatter remain intriguing and non-intuitive even after six decades. But these ideas may also have direct application. Diffuse transport is important in structural vibrations and room acoustics and quantum dots. Rooms and structures with approximate axisymmetry, or other kinds of weakly coupled substructures that may localize, are not uncommon. Dynamical Localization is observed in lasing microcavities [68].

A further potential application of these ideas is to elastic waves in the earth. Seismic records below about $3 \mathrm{mHz}$ have corroborated theory for the normal modes of an idealized earth and given information on its large-scale structure [69]. At higher 
frequencies, however, the earth's normal modes are not resolvable, because dissipation is too great and/or modal density is too high, but analyses of the kind presented here might permit retrieval of statistical information on large scale earth structure at higher frequencies. One envisions, for example, detection of an enhancement of the diffuse field following a large earthquake, an enhancement in the vicinity of the earthquake and at its antipode, relative to the amplitude at points 90 degrees from the earthquake source. One further envisions that the enhancement would then diminish with time as the waves scattered off of large scale features that break the approximate axisymmetry of the earth around an axis going through the source and its antipode. Many of the parameters governing such measurements in the earth will be very different from those of an ultrasonics laboratory. Quality factors in the earth are less than those of the aluminum samples used here, thus limiting the time period over which diffuse seismic coda will be discernable. On the other hand, the earth permits absolute measurements of PSD (especially at low frequencies where site amplification factors are relatively uniform), without the complications encountered here due to unknown sensor sensitivities. Contact ultrasonic sensors disturb a wave field, artificially breaking symmetries and contributing inhomogeneous absorption; seismic sensors do not. The use of multiple ultrasonic contact sensors is contraindicated by their intrusiveness; the use of multiple seismic sensors is not.

This work was supported in part by grant number EAR 12-15824 from the US National Science Foundation. The authors are grateful to Thomas Neulinger for preliminary laboratory set-up and early measurements. RW would especially like to acknowledge and thank the ever-gracious Roger Maynard for stimulating conversations. He will long be remembered.

\section{References}

1. C.B. Guo, P. Holler, K. Goebbels, Scattering of Ultrasonic waves in anisotropic polycrystalline metals, Acustica 59, 112 (1985)

2. P. Anugonda, J.S. Wiehn, J.A. Turner, Diffusion of ultrasound in concrete, Ultrasonics 39, 429 (2001)

3. J. Becker, L.J. Jacobs, J. Qu, Characterization of cement-based materials using diffuse ultrasound, J. Eng. Mech. 129, 1478 (2003)

4. R. Weaver, W. Sachse, Diffusion of ultrasound in a glass bead slurry, J. Acoust. Soc. Am. 97, 2094 (1995)

5. R. Weaver, Diffusivity of Ultrasound in Polycrystals, J. Mech. Phys. Solids 38, 55 (1990)

6. Y. Lu, J. Michaels, A methodology for structural health monitoring with diffuse ultrasonic waves in the presence of temperature variations, Ultrasonics 43, 717 (2005)

7. R.B. Thompson, F.J. Margetan, P.L.Y. Haldipur, A. Li, P. Panetta, H. Wasan, Scattering of elastic waves in simple and complex polycrystals, Wave Motion 45, 655 (2008)

8. S.K. Ramamoorthy, Y. Kane, J.A. Turner, Crack Depth Determination in Concrete by Ultrasound Diffusion, J. Acoust. Soc. Am. 115, 523 (2004)

9. Y. Zhang, T. Planes, E. Larose, A. Obermann, C. Rospares, G. Moreau, Diffuse ultrasound monitoring of stress and damage development on a 15-ton concrete beam, J. Acoust. Soc. Am. 139, 1691 (2016)

10. R. Hennino, N. Trégourès, N.M. Shapiro, L. Margerin, M. Campillo, B.A. van Tiggelen, R.L. Weaver, Observation of equipartition of seismic waves, Phys. Rev. Lett. 86, 3447 (2001)

11. M. Campillo, A. Paul, Long-range correlations in the diffuse seismic coda, Science 299, 547 (2003) 
12. N.M. Shapiro, M. Campillo, L. Stehly, M.H. Ritzwoller, High-resolution surface-wave tomography from ambient seismic noise, Science 307, 1615 (2005)

13. P. Gouedard, L. Stehly, F. Brenguier, M. Campillo, Y. Colin de Verdière, E. Larose, L. Margerin, P. Roux, F.J. Sánchez-Sesma, N.M. Shapiro, R.L. Weaver, Cross-correlation of random fields: mathematical approach and applications, Geophys. Prospecting 56, 375 (2008)

14. E. Larose, L. Margerin, B.A.van Tiggelen, M. Campillo, Weak localization of seismic waves, Phys. Rev. Lett. 93, 048501 (2004)

15. F.J. Sánchez-Sesma, M. Rodríguez, U. Iturrarán-Viveros, F. Luzón, M. Campillo, L. Margerin, A. García-Jerez, M. Suarez, M.A. Santoyo, A. Rodríguez-Castellanos, A theory for microtremor $H / V$ spectral ratio: application for a layered medium, Geophys. J. Inter. 186, 221 (2011)

16. T. Maeda, H. Sato, M. Ohtake, Constituents of vertical-component coda waves at long periods, Pure Appl. Geophys. 163, 549 (2006)

17. R.L. Weaver, O.I. Lobkis, Anderson Localization in Coupled Reverberation Rooms J. Sound. Vibr. 231, 1111 (2000)

18. J. de Rosny, A. Tourin, M. Fink, Coherent backscattering of an elastic wave in a chaotic cavity, Phys. Rev. Lett. 84, 1693 (2000)

19. R.L. Weaver, O.I. Lobkis, Enhanced backscattering and modal echo of reverberant elastic waves, Phys. Rev. Lett. 84, 4942 (2000)

20. R.L. Weaver, Anderson localization of ultrasound, Wave Motion 12, 129 (1990)

21. H. Hu, A. Strybulevych, J.H. Page, S.E. Skipetrov, B.A. van Tiggelen, Localization of ultrasound in a three-dimensional elastic network, Nat. Phys. 4, 945 (2008)

22. R.L. Weaver, Spectral statistics in elastodynamics, J. Acoust. Soc. Am. 85, 1005 (1989)

23. C. Ellegaard, T. Guhr, K. Lindemann, J. Nygård, M. Oxborrow, Symmetry breaking and spectral statistics of acoustic resonances in quartz blocks, Phys. Rev. Lett. 77, 4918 (1996)

24. O.I. Lobkis, I.S. Rozhkov, R.L. Weaver, Nonexponential dissipation in a lossy elastodynamic billiard: Comparison with Porter-Thomas and random matrix predictions, Phys. Rev. Lett. 91, 194101 (2003)

25. T. Gorin, T.H. Seligman, R.L. Weaver, Scattering fidelity in elastodynamics, Phys. Rev. E 73, $015202(2006)$

26. O.I. Lobkis, R.L. Weaver, Coda-wave interferometry in finite solids: Recovery of P-to-S conversion rates in an elastodynamic billiard, Phys. Rev. Lett. 90, 254302 (2003)

27. R.L. Weaver, On diffuse waves in solid media, J. Acoust. Soc. Am. 71, 1608 (1982)

28. G. Tanner, N. Sondergaaard, Wave chaos in acoustics and elasticity, J. Phys. A Math. Theor. 40, R443 (2007)

29. N. Sondergaard, G. Tanner, Wave chaos in the elastic disk, Phys. Rev. E 66, 066211 (2002)

30. H.-J. Stöckmann, Quantum Chaos: An Introduction (Cambridge University Press, 1999) ISBN 0-521-59284-4

31. R. Weaver, Wave Chaos in Elastodynamics, in Waves and Imaging through Complex Media, edited by P. Sebbah (Kluwer, Dordrecht 2001) pp. 141-186

32. R.H. Lyon, R.G. DeJong, Theory and Application of Statistical Energy Analysis, 2nd edn. (Butterworth-Heineman 1995)

33. J.L. Davy, The relative variance of the transmission function of a reverberation room, J. Sound Vib. 77, 455 (1981)

34. P.J. Shorter, R.S. Langley, On the reciprocity relationship between direct field radiation and diffuse reverberant loading, J. Acoust. Soc. Am. 117, 85 (2005)

35. O.I. Lobkis, R.L. Weaver, On the emergence of the Green's function in the correlations of a diffuse field, J. Acoust. Soc. Am. 110, 3011 (2001)

36. R.L. Weaver, Ward identities and the retrieval of Green's functions in the correlations of a diffuse field, Wave Motion 45, 596 (2008)

37. P. Roux, K.G. Sabra, W.A. Kuperman, A. Roux Ambient noise cross correlation in free space: Theoretical approach, J. Acoust. Soc. Am. 117, 79 (2005) 
38. C. Sens-Schoenfelder, R. Snieder, S.C. Stahler, The lack of equipartitioning in global body wave coda, Geophys. Res. Lett. 42, 7483 (2015)

39. R.L. Weaver, O.I. Lobkis, Temperature dependence of diffuse field phase, Ultrasonics 38, 491 (2000)

40. R.L. Weaver, Diffuse elastic wave at a free surface, J. Acoust. Soc. Am. 78, 131 (1985)

41. K. Graff, Wave Motion in Elastic Solids (Dover, NY, 1975)

42. A.K. Gautesen, Scattering of a Rayleigh wave by an elastic wedge, Wave Motion 9, 51 (1987)

43. R.L. Weaver, J. Burkhardt, Weak Anderson localization and enhanced backscatter in reverberation rooms and quantum dots, J. Acoust. Soc. Am. 96, 3186 (1994)

44. D.E. Newland, An Introduction to Random Vibrations, Spectral \& Wavelet Analysis: Third Edition, Chapter 9 (Dover, NY, 2005)

45. J.R. Hutchinson, Vibrations of Solid Cylinders, J. Appl. Mech. 47, 901 (1980)

46. For a proof in English, see Strauss, Walter A. Partial Differential Equations (John Wiley \& Sons 2008), Chap. 11

47. C. Kittel, H. Kroemer, Thermal Physics, 2nd edn. (Macmillan, 1980)

48. N. Dupuis, R. Mazo, L. Onsager, Surface specific heat of an isotropic solid at low temperatures, J. Chem. Phys. 33, 1452 (1960)

49. G.N. Watson, A Treatise on the Theory of Bessel Functions, 2nd edn. (Cambridge University Press, 1966)

50. This is the Sokhotsky-Plemelj formula. See V.S. Vladimirov, Equations of Mathematical Physics (Marcel Dekker, 1971) ISBN 0-8247-1713-9

51. E.N. Economou, Green's Functions in Quantum Mechanics, 2nd edn. ( Springer, NY, 1983)

52. H. Diestel, Reciprocity calibration of microphones in a diffuse sound field, J. Acoust. Soc. Am. 33, 514 (1961)

53. P. Smith Jr., Response and radiation of structural modes excited by sound, J. Acoust. Soc. Am. 34, 640 (1962)

54. R. Weaver, On the Time and Geometry Independence of Elastodynamic Spectral Energy Density, J. Acoust. Soc. Am. 80, 1539 (1986)

55. M. Abromowitz, I. Stegun, Handbook of Mathematical Functions (Dover NY)

56. V.N. Prigodin, B.L. Altshuler, K.B. Efetov, S. Iida, Mesoscopic dynamical echo in quantum dots, Phys. Rev. Lett. 72, 546 (1994)

57. K.M. Frahm, D.L. Shepelyansky, Quantum Localization in Rough Billiards, Phys. Rev. Lett. 78, 1440 (1997)

58. L. Sirko, S. Bauch, Y. Hlushchuk, P.M. Koch, R. Blumel, M. Barth, U. Kuhl, H.J. Stockmann, Observation of dynamical localization in a rough microwave cavity, Phys. Lett. A 266, 331 (2000)

59. R. Blumel, S. Fishman, M. Griniasty, U. Smilansky, in Quantum Chaos and Statistical Nuclear Physics, Proceedings of the 2nd International Conference on Quantum Chaos, Curnevaca, Mexico, Edited by T.H. Seligman, H. Nishioka (Springer-Verlag, Heidelberg, 1986)

60. J. Grönqvist, T. Guhr, Energy localization in two chaotically coupled systems, Phys. Rev. E 71, 036214 (2005)

61. A.D. Lagendijk, B. van Tiggelen, D.S. Wiersma, 50 years of Anderson Localization, Phys. Today 62, 24 (2009)

62. R.L. Weaver, Transport and localization amongst coupled substructures, Phys. Rev. E 73, $036610(2006)$

63. M. Wright, R. Weaver (eds), New Directions in Linear Acoustics (Cambridge University Press, 2010)

64. F.E. Stanke, G.S. Kino, A unified theory for elastic wave-propagation in polycrystalline materials, J. Acoust. Soc. Am. 75, 665 (1984)

65. M. Lawniczak, M. Bialous, V. Yunko, S. Bauch, L. Sirko, Numerical and experimental studies of the elastic enhancement factor for 2D open systems, Acta Phys. Pol. A 128, 974 (2015) 
66. J. Burkhardt, Structural damage characterization using free decays, J. Sound. Vibr. 246, 483 (2001)

67. O.I. Lobkis, R.L. Weaver, I. Rozhkov, Power Variances and Decay Curvature in a Reverberant System, J. Sound. Vibr. 237, 281 (2000)

68. W. Fang, H. Cao, V.A. Podolskiy, E.E. Narimanov, Dynamical localization in microdisk lasers, Optics Express 13, 5641 (2005)

69. J.S. Resovsky, M.H. Ritzwoller, New and refined constraints on three-dimensional earth structure from normal modes below $3 \mathrm{mHz}$, J. Geophys. Research-Solid Earth 103, 783 (1998)

Open Access This is an Open Access article distributed under the terms of the Creative Commons Attribution License (http://creativecommons.org/licenses/by/4.0), which permits unrestricted use, distribution, and reproduction in any medium, provided the original work is properly cited. 